\title{
Efficacy of AAV9-mediated SCPL1 gene transfer in a mouse model of S1P lyase insufficiency syndrome
}

\author{
Piming Zhao,, ${ }^{1,2}$ Gizachew B. Tassew, ${ }^{1}$ Joanna Y. Lee, ${ }^{1}$ Babak Oskouian, ${ }^{1}$ Denise P. Muñoz, ${ }^{1}$ \\ Jeffrey B. Hodgin, ${ }^{3}$ Gordon L. Watson, ${ }^{1}$ Felicia Tang,, Jen-Yeu Wang, ${ }^{1}$ Jinghui Luo, ${ }^{3}$ Yingbao Yang, ${ }^{3}$ \\ Sarah King, ${ }^{1}$ Ronald M. Krauss, ${ }^{1}$ Nancy Keller, ${ }^{1}$ and Julie D. Saba ${ }^{1}$ \\ 'Department of Pediatrics, UCSF, San Francisco, California, USA. ${ }^{2}$ Cure Genetics, Suzhou, China. ${ }^{3}$ Department of \\ Pathology, University of Michigan, Ann Arbor, Michigan, USA.
}

\begin{abstract}
Sphingosine-1-phosphate lyase insufficiency syndrome (SPLIS) is a rare metabolic disorder caused by inactivating mutations in sphingosine-1-phosphate lyase 1 (SCPL1), which is required for the final step of sphingolipid metabolism. SPLIS features include steroid-resistant nephrotic syndrome and impairment of neurological, endocrine, and hematopoietic systems. Many affected individuals die within the first 2 years. No targeted therapy for SPLIS is available. We hypothesized that SCPL1 gene replacement would address the root cause of SPLIS, thereby serving as a universal treatment for the condition. As proof of concept, we evaluated the efficacy of adeno-associated virus 9-mediated transfer of human SCPL1 (AAV-SPL) given to newborn Sgp/1-KO mice that model SPLIS and die in the first weeks of life. Treatment dramatically prolonged survival and prevented nephrosis, neurodevelopmental delay, anemia, and hypercholesterolemia. STAT3 pathway activation and elevated proinflammatory and profibrogenic cytokines observed in KO kidneys were attenuated by treatment. Plasma and tissue sphingolipids were reduced in treated compared with untreated KO pups. SCPL1 expression and activity were measurable for at least $\mathbf{4 0}$ weeks. In summary, early AAV-SPL treatment prevents nephrosis, lipidosis, and neurological impairment in a mouse model of SPLIS. Our results suggest that SCPL1 gene replacement holds promise as a durable and universal targeted treatment for SPLIS.
\end{abstract}

Conflict of interest: PZ and JDS are inventors on a pending patent application, International Application serial number PCT/US2021/018613 titled: Adeno-Associated Viral (Aav)-Mediated Sgpl1 Gene Therapy For Treatment Of Sphingosine1-Phosphate Lyase Insufficiency Syndrome (Splis). This research was supported by a gift from Ultragenyx Pharmaceutical Inc. in January 2019 (to JDS).

Copyright: (c) 2021, Zhao et al. This is an open access article published under the terms of the Creative Commons Attribution 4.0 International License.

Submitted: November 11, 2020 Accepted: March 17, 2021

Published: March 23, 2021

Reference information: /CI Insight. 2021;6(8):e145936.

https://doi.org/10.1172/jici.

insight.145936.

\section{Introduction}

Biallelic loss-of-function mutations in sphingosine-1-phosphate lyase 1 (SGPL1) result in sphingosine-1phosphate lyase insufficiency syndrome (SPLIS), a rare metabolic disorder associated with nonlysosomal sphingolipid storage $(1,2)$. The syndrome was first described in $2017(3,4)$. About 50 cases have been reported to date (5-10). Most affected individuals exhibit steroid-resistant nephrotic syndrome (SRNS) progressing rapidly to end-stage renal disease. Nephrosis is most often associated with focal segmental glomerulosclerosis pathology, including the aggressive collapsing variant. Primary adrenal insufficiency is the second most common feature. Defects of the central and peripheral nervous systems, which may include developmental delay or regression accompanied by characteristic findings on magnetic resonance imaging, occur with other disease features or independently in about half of cases $(1,11)$. T cell lymphopenia seems to be a universal feature, although some level of $\mathrm{T}$ cell function usually persists. A wide range of severity has been observed, with some affected individuals dying in utero, and others in infancy, while still others have presented later in the first decade of life and are living into adulthood with supportive care. No specific therapies for the treatment of SPLIS have been established.

SGPL1 encodes sphingosine phosphate lyase (SPL), the enzyme responsible for the final step of sphingolipid metabolism (12). SPL catalyzes the irreversible degradation of phosphorylated sphingoid bases, generating 2 products: a long chain aldehyde and ethanolamine phosphate. The bioactive sphingolipid sphingosine-1-phosphate (S1P) is the major SPL substrate. S1P is the ligand for G protein-coupled S1P receptors (S1PRs) involved in the control of actin cytoskeletal organization, cell migration, and cell survival (13). S1P signaling regulates lymphocyte trafficking, angiogenesis, inflammation, and other physiological processes (14). SPL inactivation in vivo causes profound tissue S1P elevation and accumulation of upstream sphingolipid intermediates, such 
as ceramide and sphingosine, which have cytotoxic properties (15). SPL inactivation disrupts S1P chemotactic gradients needed for lymphocyte egress from lymphoid tissues, which explains why individuals with SPLIS are lymphopenic $(16,17)$. The mechanisms responsible for the pathological impact of SPL inactivation on organ functions could be due to aberrant S1P signaling, intracellular effects of S1P, accumulation of cytotoxic sphingolipids, deficiency of SPL products, broader disruption of lipid homeostasis, or any combination of these.

SPL localizes to the outer membrane of the endoplasmic reticulum (18). In contrast to conventional sphingolipidoses - which are usually lysosomal lipid storage conditions - SPLIS shows no evidence of lysosomal lipid storage. The first SPLIS cases were identified by next-generation sequencing (NGS). Individuals with SPLIS have since been identified by NGS or disease-focused diagnostic genetic panels. Pathogenic variants including missense, nonsense, and splice site mutations affecting 14 of the 15 SGPL1 exons have been reported (19).

We hypothesized that virus-mediated gene replacement may afford a practical strategy to treat and potentially cure SPLIS by specifically addressing its root cause. Adeno-associated virus (AAV) is a small, single-stranded DNA virus belonging to the parvovirus family (20). AAV vectors are nonintegrating, form episomal concatemers, have relatively low immunogenicity, and can persist in postmitotic tissues for years $(21,22)$. Twelve serotypes are recognized based on neutralizing antibodies against capsid proteins that determine tissue tropism (22). Safety of AAV-based gene therapy has been established in more than 170 clinical trials, with proven efficacy for several diseases. In 2019, the FDA approved Zolgensma, the first systemic, AAV-mediated gene therapy for spinal muscular atrophy (23).

A constitutive Sgpl1-KO mouse generated by gene trapping represents the most well-established murine model of SPLIS (24). Sgpl1-KO pups are born at 25\% frequency from heterozygous mating pairs. Sgpl1-KO pups lack SPL expression and activity and accumulate sphingolipids (15). They exhibit runting at about a week of life accompanied by anemia and lymphopenia and die at the time of weaning (around 3 weeks of age). They develop nephrosis associated with hypoalbuminemia and elevated urine albumin/creatinine ratio (ACR) (25). Histological examination of kidney sections revealed enlarged glomeruli and ultrastructural evidence of podocyte foot process effacement $(3,25)$. Subtle abnormalities of adrenal gland histology have been observed (4). Overt neurological defects have not been reported in Sgpl1-KO mice; however, brain-specific Sgpl1-KO mice that survive to adulthood exhibit behavioral and motor functional deficits (26). Thus, Sgpl1$\mathrm{KO}$ mice recapitulate many biochemical and phenotypic features of SPLIS. Other features of the KO include high liver and plasma cholesterol, glycerophospholipids, and Th-17 pathway activation (15, 27). Heterozygous Sgpl1-null mice are indistinguishable from WT in growth, survival, and sphingolipid levels. Further, transgenic mice with $10 \%-20 \%$ of WT SPL levels are lymphopenic but otherwise healthy, live normal life spans, and do not exhibit the severe phenotypes found in Sgpl1-KO mice (28). Thus, a modest level of SPL expression may prevent most SPLIS-related pathologies.

In this study, we used AAV9-mediated gene delivery to transfer the WT human SGPL1 cDNA to the tissues of Sgpl1-KO mice within the first days of life (DOL). Our baseline studies reveal for the first time to our knowledge that Sgpl1-KO mice exhibit delayed acquisition of neurodevelopmental milestones and are deficient in corticosterone, the main glucocorticoid found in mice. We demonstrate that SGPL1 gene replacement prevents nephrosis, developmental delay, and lipidosis in Sgpl1-KO mice and dramatically prolongs their survival. Further, we observed elevated proinflammatory and profibrogenic cytokines and activation of the STAT3 signaling pathway in untreated KO kidneys, all of which were attenuated by treatment. Our cumulative results provide proof of concept for AAV-mediated SGPL1 gene transfer as a potentially curative therapy for SPLIS and provide insight into the possible pathomechanism of SPLIS nephrosis.

\section{Results}

$A A V$-SPL restores SPL expression and activity in SPLIS patient-derived fibroblasts. Human WT SGPL1 cDNA or a self-cleaving bicistronic system for coexpressing red fluorescent protein (RFP) and SPL were cloned in an AAV2 vector under control of the CMV promoter. The resulting constructs, AAV-SPL and AAV-SPLtRFP, were amplified, packaged in AAV8 capsid, and used to transduce SPLIS patient-derived fibroblasts, which exhibit low SPL expression and activity (10). Transduction of fibroblasts with AAV-SPL increased SPL expression (Figure 1A) and activity (Figure 1B). Fibroblasts transduced with AAV-SPL-tRFP resulted in lower expression and activity. Based on these results, AAV-SPL was chosen for validation in vivo. A construct expressing an SPL that harbors a missense mutation at lysine 353 (AAV-SPL ${ }^{\mathrm{K} 353 \mathrm{~L}}$ ) — which is the site for pyridoxal 5'-phosphate cofactor binding and was shown to completely eliminate enzyme function - was generated to serve as a biochemical control. AAV9 was selected for in vivo studies based on its 
A

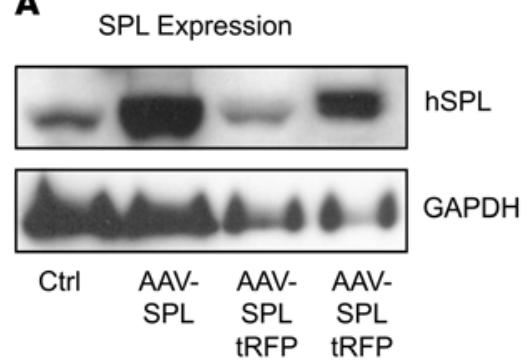

$(5 X)$

B

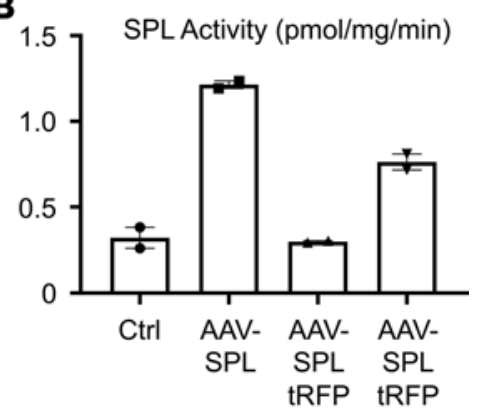

$(5 X)$

Figure 1. AAV-SPL expression and activity in vitro. The human SPL (hSPL) CDNA and the hSPL-tRFP cDNA were cloned into pAAV-MCS, packaged in AAV8, and used to transduce SPLIS skin fibroblasts. (A) Immunoblot of hSPL in whole cell extracts of SPLIS fibroblasts treated with vehicle (Ctrl), AAV-SPL, an equal volume of AAV-SPLtRFP, or a 5 -fold higher volume of AAV-SPL-tRFP. (B) SPL activity in extracts corresponding to the samples described in $\mathbf{A}$.

broad tropism, including brain, adrenal gland, and kidney — all of which are involved in SPLIS — as well as liver, a major site of metabolism of blood sphingolipids.

Treatment of Sgpl1-KO mice with AAV-SPL dramatically prolongs survival. To test the impact of SGPL1 gene replacement on Sgpl1-KO mouse survival, AAV-SPL was delivered to 10 newborn Sgpl1-KO pups using the strategy shown in Figure 2A. Litters produced from heterozygous matings were genotyped on DOL 1 by toe biopsy. Sgpll-KO pups were treated at 1-2 DOL with a single dose of approximately $7 \times 10^{11}$ vector genomes (vg) by intravenous (i.v.) injection. An additional $3 \mathrm{KO}$ pups were injected with the same dose of AAV-SPL ${ }^{\text {K333L }}$. Pups were monitored for weight gain and euthanized when the humane endpoint was reached. As shown in Figure 2B, AAV-SPL-treated Sgpl1-KO mice were appreciably larger than untreated Sgpl1-KO mice. Sgpl1-KO mice treated with AAV-SPL gained weight steadily, as shown in Figure 2C. Mean survival increased from 11 \pm 7 days in untreated KO controls to $137 \pm 114$ days in treated mice ( $n=16$ untreated, $n=10$ treated; $P<$ 0.0002 ), with some treated mice living for 8-11.5 months (Figure 2D). In contrast, mice treated with catalytically inactive AAV-SPL ${ }^{\mathrm{K} 33 \mathrm{~L}}$ showed a mean survival of $12.8 \pm 6.2$ days (Figure 2D). Based on these findings, we concluded that introduction of a functional hSPL into Sgpll-KO pups significantly prolongs their survival.

AAV-SPL treatment of Sgpl1-KO mice prevents SPLIS-associated nephrosis. Renal involvement is present in $80 \%$ of SPLIS cases and is a major cause of morbidity, hospitalizations, surgical intervention (renal transplantation), and death. Similarly, Sgpll-KO mice develop nephrosis with high urine albumin/creatinine ratio (ACR) and low serum albumin levels, accompanied by pathological changes consistent with podocyte and glomerular injury prior to their demise at the time of weaning (at 21 DOL). The first few Sgpll-KO mice treated in our series lived for 35-70 days and exhibited higher serum albumin levels than untreated Sgpl1-KO mice (data not shown). This indicated that AAV-SPL treatment may have delayed the progression of nephrosis but did not completely prevent it. In contrast, once we optimized our virus delivery, we could routinely prolong survival of treated mice for 8-11.5 months. These mice showed normal ACR (Figure 3A) and serum albumin levels (Figure 3B) up to the time of their deaths. Periodic acid-Schiff-stained sections of kidney cortices from untreated Sgpll-KO mice showed enlarged glomeruli with a wide size distribution and mesangial expansion compared with WT glomeruli (Figure 3C). These features were absent in the kidney cortices of Sgpl1-KO mice treated with AAV-SPL but were present in those of Sgpl1-KO mice treated with catalytically inactive AAV-SPL ${ }^{\mathrm{K} 335 \mathrm{~L}}$ (Figure 3C). Average glomerular tuft size is shown quantitatively in Figure 3D. Some glomeruli of untreated Sgpll-KO mice and Sgpl1-KO mice treated with AAV-SPL ${ }^{\text {K333L }}$ exhibited sclerosis, an example of which is shown in Figure 3C. No glomeruli from WT and AAV-SPL-treated Sgpll-KO mice exhibited focal sclerosis.

SPLIS kidneys show Stat3 activation and cytokine upregulation. STAT3 is a transcription factor that contributes to carcinogenesis and the regulation of inflammation, autophagy, cellular metabolism, and mitochondrial function (29). It can be activated by various cytokines through binding and activation of gp130, the IL-6 receptor. Glomerular injury and S1P signaling have been associated with activation of STAT3 in mice and humans (30-34). We compared the phosphorylated (active) and total mouse Stat3 protein levels in Sgpl1 WT and KO kidney homogenates by immunoblotting. As shown in Figure 4A, Sgpll-KO kidneys exhibited activation of Stat3 in comparison with WT controls. Stat3 activation was muted in AAV-SPL-treated KO kidneys, whereas kidneys of $\mathrm{KO}$ mice treated with AAV-SPL ${ }^{\mathrm{K} 335 \mathrm{~L}}$ were not protected from Stat3 activation. 
A

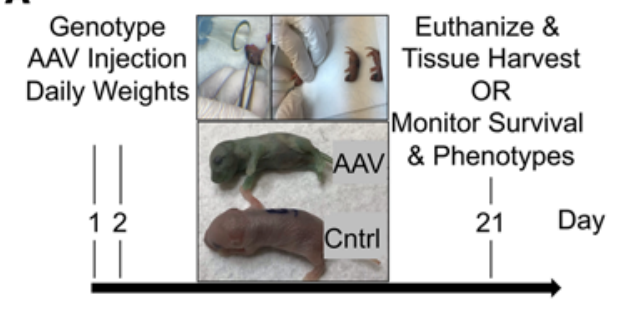

C

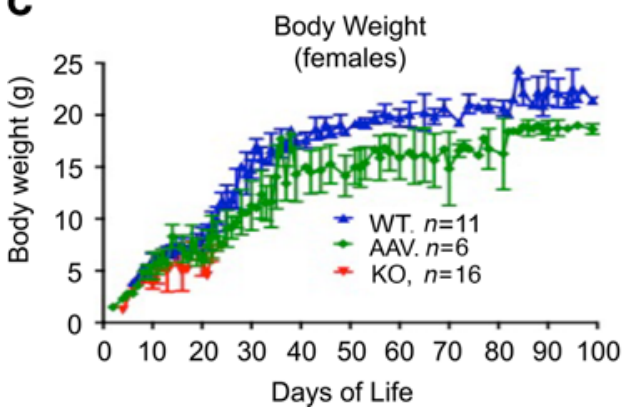

B

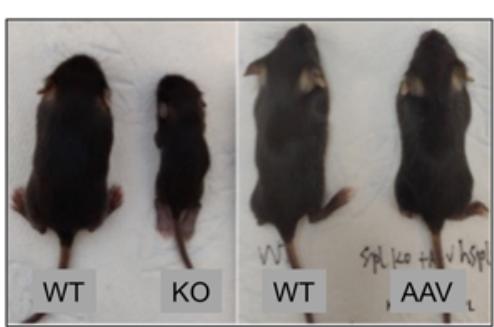

D

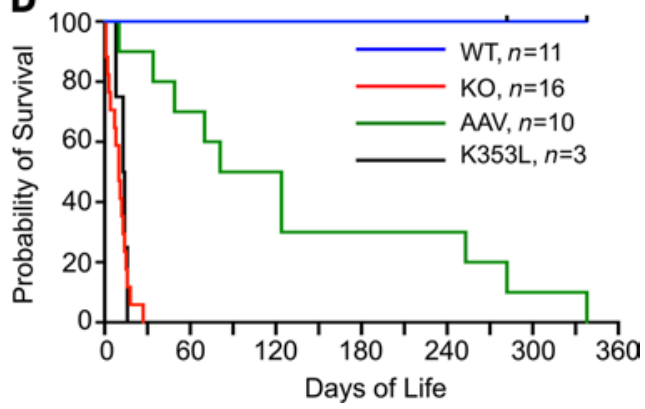

Figure 2. Treatment of newborn Sgpl1-KO mice with AAV-SPL prolongs survival. (A) Treatment schedule, with upper images showing injection site and lower image showing green color of properly injected KO pup (AAV) next to untreated WT pup (Cntrl). (B) Images showing size discrepancy between WT and untreated Sgp/7-KO littermates at 17 DOL versus similarity in size of WT and AAV-SPL-treated Sgp/7-KO (AAV) littermates at 22 DOL. (C) Weight gain of female WT (blue), untreated Sgp/1-KO (red), and AAV-SPL-treated KO (green) mice. (D) Kaplan-Meier survival curve for WT (blue), untreated Sgp/1-KO (red), AAV-SPL-treated KO (green), and AAV-SPL ${ }^{\mathrm{K} 353 \mathrm{~L}}$-treated KO (black) mice. Log-rank test with Bonferroni's correction: for WT vs. all other groups, $P<$ 0.0003 ; AAV vs. KO, $P<0.0003$; KO vs. K353L, no significant difference (NSD).

Expression of Stat3 target genes suppressor of cytokine signaling 1 and 3 (Socs1, Socs3) and 2 others implicated in various forms of kidney injury, namely lipocalin $2(L c n 2)$ and tissue inhibitor of metalloprotease 1 (Timp1), were measured by quantitative real-time PCR (RT-PCR) in kidney tissues from each group. Stat3 target genes were upregulated in untreated Sgpl1-KO kidney tissue compared with WT, while kidneys of AAV-SPL-treated KO mice showed lower Stat3 target gene elevation compared with untreated Sgpl1-KO mice (Figure 4B). Proinflammatory cytokine elevation in the liver tissues of Sgpl1-KO mice was previously reported (27). We confirmed that the proinflammatory cytokine and STAT3 pathway activator Il-6 was upregulated in Sgpl1-KO liver tissues (Figure 4C). Additional proinflammatory cytokines, including Tnf- $\alpha$, Ifn- $\gamma, I l-1 \beta$, and monocyte chemoattractant protein 1 (Mcp1), were elevated in Sgpl1-KO liver compared with WT liver (Figure 4C). The cytokine Tgf- $\beta$ is a well-characterized inducer of fibrosis. Tgf- $\beta$ levels were also elevated in Sgpl1-KO liver compared with WT liver (Figure 4C). Importantly, each of these cytokines was also elevated in the kidneys of the Sgpl1-KO mice compared with WT (Figure 4D). Further, AAV-SPL treatment diminished cytokine levels in Sgpl1-KO kidneys (Figure 4D). These findings demonstrate that AAVSPL treatment prevents the development of nephrosis, Stat3 activation, and proinflammatory and fibrogenic cytokine upregulation in various tissues of Sgpl1-KO mice.

$A A V-S P L$ treatment prevents neurodevelopmental delay in preweaned Sgpl1-KO mice. In nearly half of SPLIS cases, neurological manifestations are present, including sensorineural hearing loss, cranial nerve defects, a Charcot-Marie-Tooth-type peripheral neuropathy, seizures, ataxia, and developmental delay or regression. Brain-specific conditional Sgpl1-KO mice live normal life spans but exhibit motor and behavioral abnormalities as adults (26). However, characterization of the neurodevelopmental function of global Sgpl1-KO pups has not been reported to our knowledge. A battery of tests designed to measure the achievement of basic neurodevelopmental milestones in preweaned mice was used to compare AAV-SPL-treated and untreated Sgpl1-KO pups and WT littermates (Figure 5, A-F). Sgpl1-KO pups showed significant delays in the achievement of eye opening, hearing onset, and adult pattern walking. In addition, grip strength was weaker in Sgpl1-KO compared with WT pups, indicating a motor function deficit. Cliff aversion reflex scores were lower in KO pups compared with WT littermates, but the difference was not statistically significant. Other tests, including paw grasping reflex, righting reflex, negative geotaxis, and inverted clinging obstacle tests, were either highly variable from day to day or did not reveal defects in the Sgpl1-KO pups (data not shown). In contrast, the neurodevelopmental scores in AAV-SPL-treated KO pups were not significantly different from WT (Figure 5, A-F). 
A

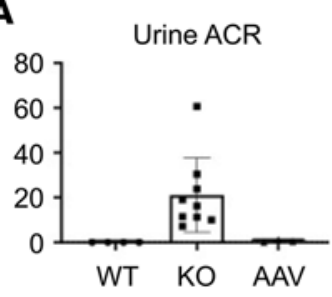

B

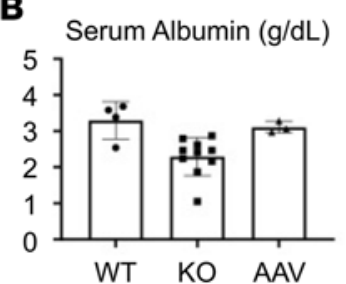

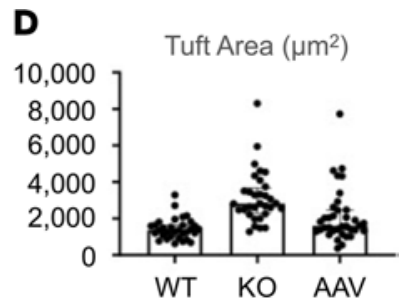

C

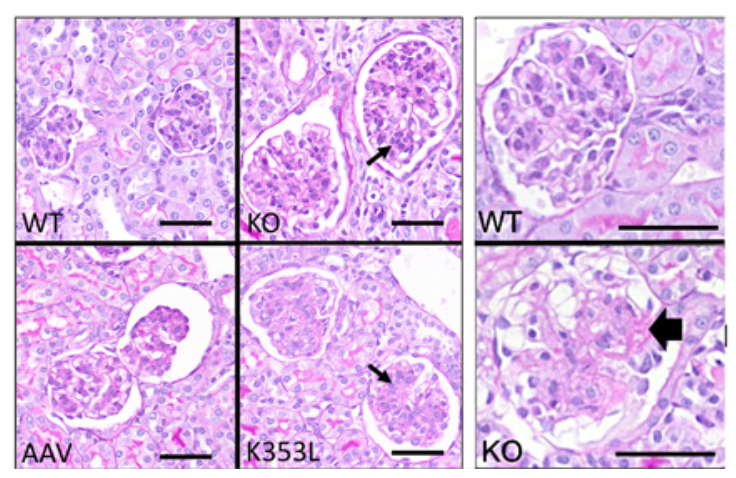

Figure 3. AAV-SPL treatment prevents development of SPLIS nephrosis. (A) Urine ACR in WT $(n=4)$, KO $(n=9)$, and AAV-SPL-treated KO (AAV, $n=3$ ) mice. (B) Serum albumin in WT $(n=4)$, KO $(n=10)$, and AAV-SPL-treated KO $(n=3)$ mice. For $\mathbf{A}$ and $\mathbf{B}$, unpaired $t$ test was performed with Welch's correction when appropriate and Bonferroni's corrections for comparisons of experimental groups with WT. For $\mathbf{A}, P=0.01$ for KO vs. WT. For $\mathbf{B}, P=0.015$. For $\mathbf{A}$ and $\mathbf{B}$, there was NSD between AAV vs. WT. (C) Quadrants show kidney sections stained with periodic acid-Schiff from WT, KO, AAV-SPL-treated KO (AAV), and AAV-SPL ${ }^{\mathrm{K} 353 \mathrm{~L}}$-treated $\mathrm{KO}$ (K353L) mice. $\mathrm{KO}$ and $\mathrm{K} 353 \mathrm{~L}$ kidney sections show enlarged glomeruli with mesangial expansion, not seen in sections of WT and AAV mice. Small arrows indicate mesangial expansion by cells and matrix. White areas surrounding glomeruli are fixation artifact. Scale bar: $50 \mu \mathrm{m}$. Image to right shows sclerosis in $\mathrm{KO}$ glomerulus (black arrowhead), with a WT glomerulus shown above it for contrast. (D) Glomerular tuft area, which includes the capillaries, mesangium, and podocytes and not Bowman's space or capsule. Mann-Whitney $U$ test was performed with Bonferroni's correction. For KO vs. WT, $P<0.0002$. There was NSD between AAV vs. WT.

We infer from these results that neurological impairment is manifest in Sgpl1-KO mice as early as the first weeks of life and that immediate treatment after birth with AAV-SPL prevents neurodevelopmental delay.

Sgpl1-KO mice exhibit glucocorticoid deficiency unresponsive to AAV-SPL. Approximately $70 \%$ of patients with SPLIS exhibit glucocorticoid hormone deficiency. However, blood steroid hormone measurements in Sgpl1-KO mice have not been hitherto reported to our knowledge. Plasma levels of corticosterone, the main glucocorticoid hormone in mice, were measured in treated and untreated Sgpll-KO mice and WT littermate controls. Samples were collected at the corticosterone peak of the circadian cycle. As shown in Figure 6A, Sgpll-KO mice exhibited significantly lower corticosterone levels than WT levels regardless of AAV-SPL treatment. Thus, Sgpll-KO mice exhibit adrenal insufficiency, which appears refractory to treatment. To determine whether low corticosterone levels in Sgpll-KO mice resulted from disruption of the hypothalamic-pituitary-adrenal axis, we measured plasma levels of the pituitary hormone adrenocorticotropic hormone (ACTH). As shown in Figure 6B, there was a trend toward lower ACTH levels in the KO compared with WT groups, with a restoration of ACTH levels in the AAV-SPL-treated KO group. However, the differences between groups were not significant. To gain additional insight into the impact of AAV-SPL on adrenal gland function, we measured the gene expression of Cyp11b1, Cyp11b2, and Akr1b7 - which respectively encode the 3 adrenal cortical enzymes 11- $\beta$-hydroxylase, aldosterone synthase, and aldo-keto-reductase - in the adrenal tissues of treated and untreated Sgpl1-KO and WT littermate mice (Figure 6, C-E). Cyp11b1 and Cyp11b2 are involved in adrenal hormone synthesis (35), whereas Akr1b7 is involved in lipid peroxide detoxification (36). Interestingly, all 3 genes were upregulated in untreated Sgpl1KO adrenal glands, whereas levels were either suppressed (Cyp11b1, Cyp11b2) or normalized compared with WT mice in the adrenal glands of AAV-SPL-treated KO mice. We infer from these findings that Sgpll-KO mice exhibit adrenal insufficiency of unclear origin with reactive upregulation of cortical gene expression. SGPL1 gene therapy reduces adrenal cortical gene expression to WT levels or below but does not restore normal corticosterone levels under the conditions tested. 
A
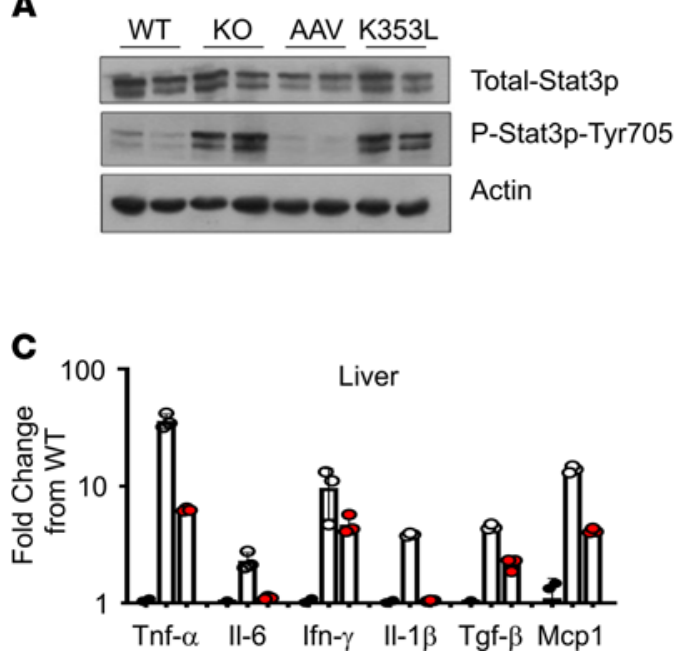

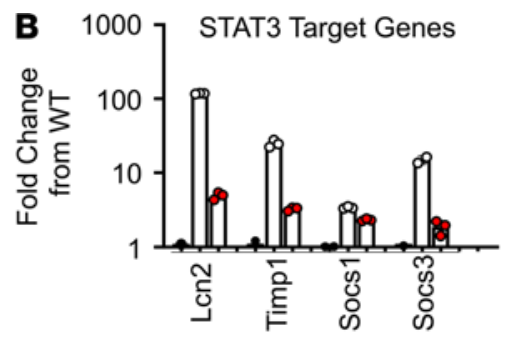

D

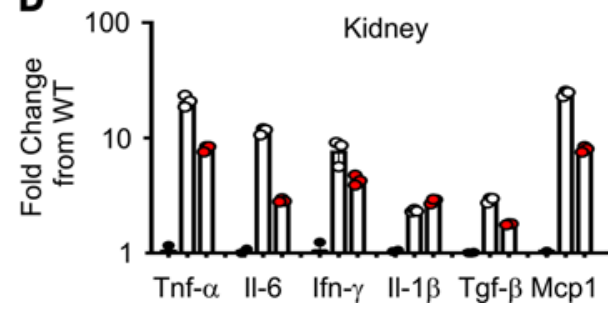

Figure 4. Stat3 activation and cytokine upregulation in SPLIS kidneys. (A) Immunoblot showing total and tyrosine

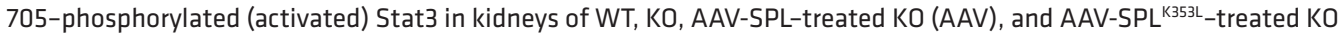
(K353L) mice; $n=2$ /group. GAPDH is a loading control. (B) Relative expression of Stat3 target genes Lcn2, Timp1, and Socs1 and Socs3 in WT (black circles), KO (white circles), and AAV kidney (red circles), shown as fold change from WT. For KO vs. AAV, $P=0.003$ for all genes except Socs3. (C) Liver and (D) kidney cytokines of WT, KO, and AAV mice, shown as log fold change from WT, with same key as in B. For B-D, unpaired $t$ test with Bonferroni's correction (and Welch's correction where appropriate) was applied. For liver cytokines, AAV vs. KO: $P=0.0097$ for Tnf- $\alpha$; $P=0.0363$ for $I I-6$; NSD for Ifn- $\gamma ; P=0.0004$ for II-1 $\beta ; P=0.0003$ for Tgf- $\beta ; P<0.0001$ for Mcp1. For kidney cytokines, AAV vs. K0: $P=0.0009$ for Tnf- $\alpha ; P=0.0023$ for II-6; $P=0.036$ for Ifn- $\gamma ; P=0.0046$ for II-1 $\beta ; P=0.005$ for Tgf- $\beta ; P<0.0001$ for Mcp1.

AAV-SPL treatment corrects anemia and hypercholesterolemia in Sgpl1-KO mice. Peripheral blood counts in Sgpl1-KO mice revealed absolute lymphopenia and anemia as shown by low hemoglobin, hematocrit, and RBC mass compared with WT (Figure 7). In contrast, AAV-SPL-treated mice were not anemic, whereas lymphopenia persisted despite AAV-SPL treatment.

Sgpl1-KO mice were previously shown to exhibit high circulating cholesterol levels, including total, free, and esterified cholesterol (15). LDL- and HDL-cholesterol were also substantially increased in KO compared with WT blood. Comparing plasma of WT, HET, KO, and AAV-SPL-treated KO mice at the time of weaning, we confirmed significantly higher levels of total cholesterol, as well as higher HDL- and non-HDL-cholesterol in KO plasma compared with WT or HET plasma, whereas triglyceride levels were not appreciably different in any of the groups (Figure 8). AAV-SPL-treated mouse plasma had total cholesterol, HDL-cholesterol, and non-HDL-cholesterol levels that were no different from WT and HET controls. Therefore, the impact of SPL disruption on circulating cholesterol was completely prevented by SGPL1 gene replacement.

Results of tests of glucose, electrolytes, and markers of liver and kidney function in the plasma of WT, $\mathrm{KO}$, and AAV-SPL-treated KO mice are shown in Table 1. Normal ranges provided for BALB/c mice by the reference laboratory served as rough guidelines, considering our study utilized mice in a C57BL/6 background. Sgpl1-KO mice exhibited normal creatinine but low albumin and high blood urea nitrogen levels, which were corrected or partially corrected with treatment. Total bilirubin and alanine transaminase levels were within normal ranges and not significantly different among the groups. Aspartate transaminase levels were within normal limits, although untreated $\mathrm{KO}$ mice had significantly higher levels than WT. Interestingly, KO mice exhibited low glucose levels, consistent with the glucocorticoid deficiency observed in these mice. Glucose levels normalized with treatment in the KO group. The only abnormality that correlated specifically with AAV-SPL treatment was a high chloride level. The significance of this is not known but could theoretically be related to metabolic acidosis that might result from deficient steroid production in treated $\mathrm{KO}$ mice.

To determine whether AAV-SPL altered S1P gradients and thereby disrupted lymphocyte trafficking in healthy mice, treatment was administered to 3 WT newborn mice. Plasma S1P levels and whole blood counts were measured at 3 months of age. The difference in plasma S1P levels between untreated (563 \pm $244 \mathrm{nM})$ and treated $(588 \pm 168 \mathrm{nM})$ WT mice was not significant. As shown in Supplemental Table 2 (supplemental material available online with this article; https://doi.org/10.1172/jci.insight.145936DS1), treated and untreated WT mice also did not exhibit significant differences in any hematological parameter. 
A

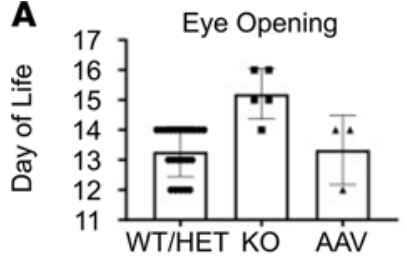

B
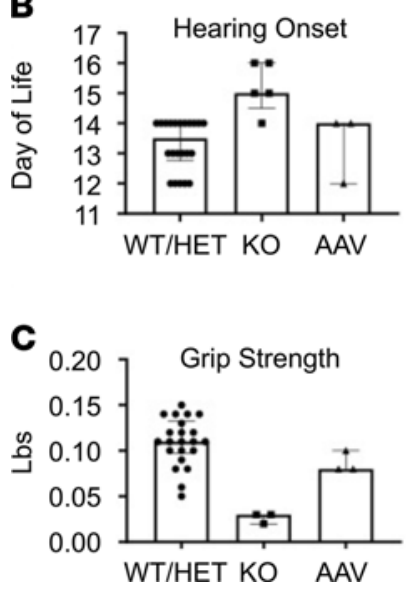

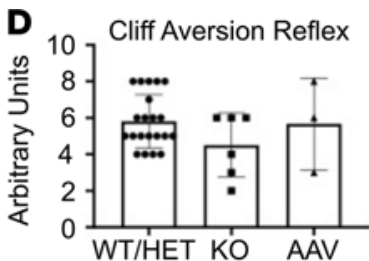

E
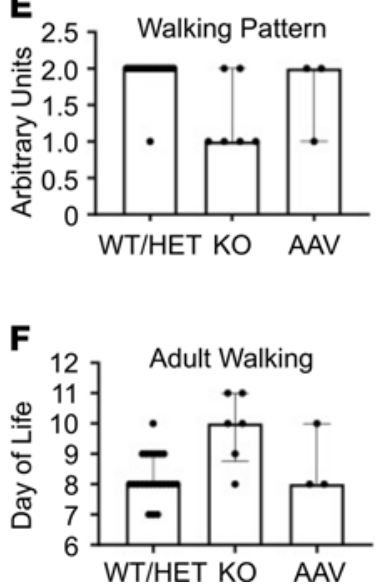

Figure 5. Sgp/1-KO mice exhibit developmental delay, which is prevented by AAV-SPL. Six neurodevelopmental milestones were scored in WT $(n=8)$, heterozygous (HET, $n=14), \mathrm{KO}(n=6)$, and AAV-SPL-treated KO (AAV, $n=3$ ) pups. For $\mathbf{A}$ and $\mathbf{D}$, unpaired $t$ test with Bonferroni's correction was applied. For B, C, and F, Mann-Whitney $U$ with Bonferroni's correction was applied. For KO vs. WT comparisons: (A, $P<0.0002)$; (B $P=0.001) ;(\mathbf{C}, P=0.0008) ;(\mathbf{D}$, NSD; E, $P=0.0068) ;(\mathbf{F}, P=0.0056)$. There was NSD between AAV and WT/HET or WT and HET for A-F.

AAV-SPL is expressed and active in key target tissues. The pattern of AAV-SPL bioavailability was investigated in Sgpl1 WT, KO, and AAV-SPL-treated KO mice. In some cases, AAV-SPL ${ }^{\mathrm{K} 353 \mathrm{~L}}$-treated KO mice were also included for comparison. Mice were euthanized for tissue harvest at 3 weeks of life unless otherwise stated.

Using quantitative RT-PCR, we compared SGPL1 expression levels in a broad range of tissues. Consistent with the known tropism of AAV9, hSPL (SGPL1) expression was high in heart and skeletal muscle, liver, and lung and lowest in spleen, kidney, and intestines (Figure 9A). In contrast, endogenous mouse SPL (mSPL, Sgpl1) expression was highest in thymus and intestine; moderate in kidney, brain, adrenal gland, and liver; and lowest in lung, spleen, and muscle, similar to our previously reported findings (data not shown and ref. 37). Representing the expression of the transgene relative to endogenous expression as a ratio, the highest levels were observed in heart, muscle, liver, and brain and lowest in thymus, intestine, and spleen (Figure 9B). Relative transgene expression in kidney, adrenal gland, brain, and liver were calculated to be $17 \%$, 2-fold, 8-fold, and 35-fold of endogenous mSPL expression levels, respectively. As shown in Figure 9C, SPL activity levels in AAV-SPL-treated KO brain, kidney, and liver were comparable to or above WT levels, ranging from 3 to $6 \mathrm{pmol} / \mathrm{mg} / \mathrm{min}$ in brain, 24 to $32 \mathrm{pmol} / \mathrm{mg} / \mathrm{min}$ in kidney, and 28 to $87 \mathrm{pmol} / \mathrm{mg} / \mathrm{min}$ in liver. SPL activity in untreated $\mathrm{KO}$ and AAV-SPL ${ }^{\mathrm{K} 353 \mathrm{~L}}$-treated $\mathrm{KO}$ tissues ranged from 0.17 to $2.3 \mathrm{pmol} / \mathrm{mg} /$ min. Adrenal gland SPL activity could not be measured because of the limited amount of available tissue.

To further confirm our findings and characterize regional transgene expression patterns within key organs affected by SPLIS, IHC was performed on fixed brain, adrenal gland, kidney, and liver sections from WT and

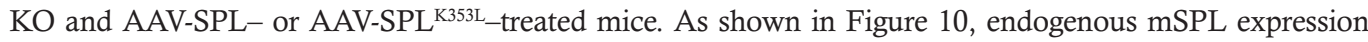
was detectable in all 4 WT tissues, whereas no signal was detected in Sgpl1-KO tissues. In liver, mSPL expression was relatively homogeneous. In other tissues, specific cell types expressed high mSPL levels, including medullary cells of the adrenal gland, Purkinje cells of the cerebellum, and both tubular and glomerular cells in the kidney. In AAV-SPL-treated Sgpl1-KO mouse tissues, strong hSPL signal was detected in all 4 tissues, although in most cases the patterns were dissimilar to endogenous mSPL expression patterns, consistent with the fact that the transgene is under regulation of the CMV promoter and not the endogenous Sgpl1 promoter. The expression pattern of hSPL in the brain was unique among the 4 tissues in that it mirrored that of the endogenous mSPL expression pattern, which was strikingly positive in Purkinje cells. This is likely a fortuitous coincidence related to the transduction pattern associated with i.v. delivery of AAV9 to pups (38). To improve delivery of AAV-SPL to brain, we packaged the virus in PHP.eB, a designer capsid with reportedly enhanced neuronal targeting capacity (39). The brain tissue of Sgpl1-KO mice treated with AAV-SPL packaged in PHP.eB showed a similar pattern of Purkinje cell targeting that appeared more intense than that 
A Plasma Corticosterone $(\mathrm{ng} / \mathrm{mL})$

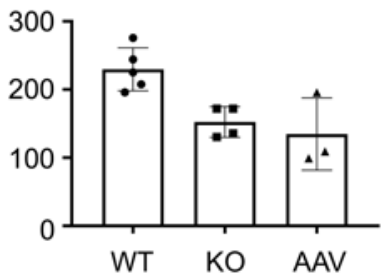

B Plasma $\mathrm{ACTH}$

$(\mathrm{ng} / \mathrm{mL})$

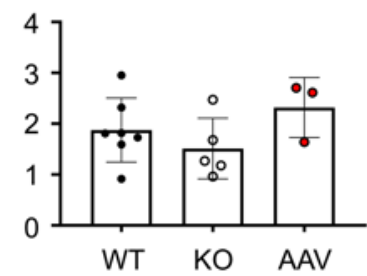

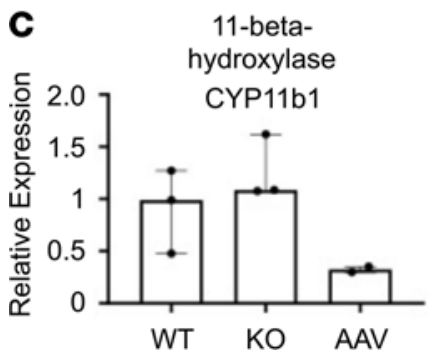
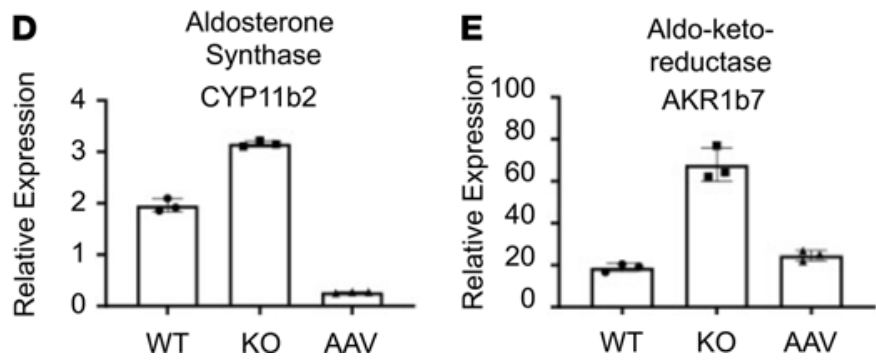

Figure 6. Sgp/1-KO mice exhibit glucocorticoid deficiency. (A) Corticosterone was measured in plasma of WT $(n=5)$, KO ( $n$ $=4)$, and AAV-SPL-treated KO (AAV, $n=3)$ mice. (B) ACTH levels were measured in the plasma of WT $(n=8), K O(n=5)$, and AAV-SPL-treated KO (AAV, $n=3$ ) mice. There are NSDs among the groups. (C-E) Expression levels of Cyp11b1, Cyp11b2, and Akr1b7 were measured in adrenal gland tissues of WT, KO, and AAV mice $(n=3$ /group except for AAV in $\mathbf{C}$, where $n=2)$. For A, C, and D, unpaired $t$ test with Bonferroni's correction was performed (with Welch's correction in C). For B, Mann-Whitney $U$ was performed. In A, KO vs. WT, $P=0.009 ; A A V$ vs. WT, $P=0.035$. In B, KO vs. WT, NSD; insufficient data for AAV vs. WT. In C, KO vs. WT, $P=0.0016 ;$ AAV vs. WT, $P=0.0032$. In D, KO vs. WT, $P=0.001 ;$ AAV vs. WT, NSD.

observed in mice treated with our standard AAV9. The hSPL expression pattern in Sgpl1-KO pups treated with catalytically inactive AAV-SPL ${ }^{\mathrm{K} 333 \mathrm{~L}}$ mirrored that of AAV-SPL-treated mouse tissues, as expected.

$A A V-S P L$ reduces plasma and tissue sphingolipids in Sgpl1-KO mice. Sgpl1-KO mice accumulate tissue S1P, reaching levels up to many hundred-fold higher than their WT and heterozygous littermates, most notably in liver, intestines, and heart (Table 2). Because SPL guards the exit point of the sphingolipid degradative pathway, other sphingolipids, including unphosphorylated and phosphorylated sphingoid bases, ceramides, and dihydrosphingolipids, also accumulate in Sgpll-KO mouse tissues. When liver S1P levels were compared in untreated and AAV-SPL-treated Sgpll-KO mice, a dramatic reduction in S1P was observed in response to treatment, although levels remained higher than those of WT (Figure 11A). Similarly, the elevated levels of dihydroS1P, sphingosine, and dihydrosphingosine (Figure 11A) as well as C14, C16, and $\mathrm{C} 18$ ceramides (Supplemental Figure 1) observed in KO liver were reduced by treatment. In contrast, C20 ceramides were not elevated in untreated KO liver and remained unchanged in response to treatment. Plasma S1P levels in untreated KO mice were 7-fold higher than WT levels (Figure 11B). KO plasma S1P levels were reduced by $50 \%$ in response to treatment. Similarly, the elevated levels of plasma dihydroS1P, sphingosine, and dihydrosphingosine in untreated $\mathrm{KO}$ mice were reduced in response to treatment.

The effects of AAV-SPL are durable over time. Importantly, hSPL protein expression persisted over time as shown by the strong SPL signal in immunoblots of liver extracts harvested from AAV-SPL-treated KO mice as late as 11 months after injection (Figure 12A). SPL enzyme activity in the liver tissues of long-lived AAV-SPL-treated KO mice was at or exceeded the level in WT mouse liver, although because of the wide variability in activity, the differences between groups did not reach statistical significance (Figure 12B). These overall results demonstrate that AAV-mediated SGPL1 gene transfer results in SPL expression and activity in a broad range of murine tissues and is durable over time.

Treatment of Sgpl1-KO mice with AAV-SPL elicits an immune response. To determine whether Sgpl1-KO mice respond immunologically to AAV-SPL, ELISAs were performed to detect the presence of anti-AAV9 in the plasma (Supplemental Figure 2). In untreated Sgpl1-KO mouse plasma, anti-AAV9 antibodies were detected in the range of $150-750 \mathrm{ng} / \mathrm{mL}$, whereas treated $\mathrm{KO}$ mouse plasma had a significantly higher concentration of AAV9-specific antibodies, in the range of 2000-3500 ng/mL. Anti-hSPL antibodies trended higher in the treated group, but the difference was not significant. Overall, our results demonstrate that untreated mice 

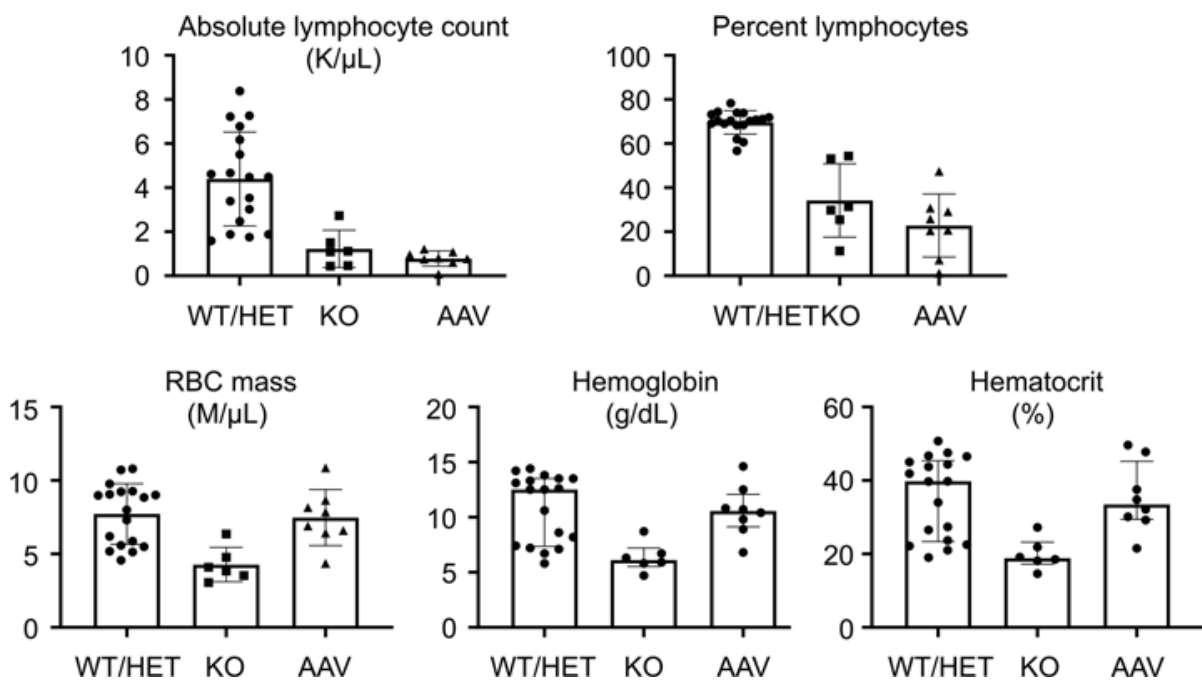

Figure 7. Hematological parameters of treated and untreated Sgp/1-KO mice. (A) Blood parameters including absolute lymphocyte count $(\mathrm{K} / \mu \mathrm{L})$, percentage lymphocytes (\% lymph), RBC mass $(\mathrm{M} / \mu \mathrm{L})$, hemoglobin $(\mathrm{g} / \mathrm{dL})$, and hematocrit $(\%)$ were measured in WT/HET $(n=18)$, KO $(n=6)$, and AAV-SPL-treated KO (AAV) mice $(n=8)$. All mice were euthanized at $28 \mathrm{DOL}$. All parameters were evaluated using unpaired 2-tailed $t$ test with Bonferroni's correction. Welch's correction was applied when appropriate. For absolute lymphocyte count, WT/HET vs. KO, $P<0.004$; AAV vs. WT/HET, $P<0.0002$. For percentage lymphocytes, WT/HET vs. KO, $P=0.006$; AAV vs. WT/HET, $P<0.0002$. For RBC mass, WT/ HET vs. KO, $P=0.0018$. For hemoglobin, WT/HET vs. KO, $P=0.0048$. For hematocrit, WT/HET vs. KO, $P=0.0026$. For RBC mass, hemoglobin and hematocrit, AAV vs. WT/HET, there was NSD.

have low but detectable amounts of anti-AAV9 antibody and that Sgpl1-KO mice have a functional immune response to the AAV vector in spite of their lymphopenia.

\section{Discussion}

SPLIS is a rare lethal childhood syndrome that was first recognized 4 years ago. Of the main disease features, SRNS leading to rapid development of end-stage renal disease and progressive neurological deterioration are responsible for most SPLIS-associated deaths, and adrenal insufficiency is also highly consequential. The characteristic lymphopenia is caused by high tissue S1P levels, which disrupt S1P chemotactic gradients critical for regulating lymphocyte egress $(16,17,40)$. With the exception of the well-established role of S1P in lymphocyte trafficking, the pathomechanisms responsible for the varied phenotypes associated with SPLIS remain poorly understood. Pathology could stem from accumulation of cellular S1P or other sphingolipids, aberrant S1PR signaling, loss of SPL products, or a combination of these.

Various targeted therapeutic approaches could be considered for treating patients with SPLIS. Some SPLIS mutations produce poorly expressed mutant SPL proteins that aggregate in cultured cells, which suggests protein misfolding and rapid clearance (3). Supplementation with high-dose vitamin $\mathrm{B}_{6}$ (which can overcome reduced affinity for the cofactor and/or exert chaperone functions for $\mathrm{B}_{6}$-dependent enzymes) (41) or chemical chaperones (42) could potentially stabilize some mutant SPL proteins. There is evidence in support of this approach (10). However, these strategies would not be as effective in patients harboring SGPL1 alleles with deletions, nonsense or catalytic site mutations, or splicing defects. Substrate depletion strategies are effective in some patients with sphingolipidoses and attenuate phenotypes observed in SPL/Sply mutant fruit flies (43-45). However, L-cycloserine - the only FDA-approved drug that blocks sphingolipid biosynthesis by inhibiting serine palmitoyltransferase - also inhibits SPL activity (Dominic Campopiano, University of Edinburgh, personal communication). Supplementation with SPL products could have a role in SPLIS treatment. However, SPL products hexadecenal and ethanolamine-1P are not FDA approved, and their deficiency is unlikely to cause all SPLIS disease manifestations. Sphingosine kinase inhibitors, S1P-binding monoclonal antibodies, and S1PR antagonists may have a place in SPLIS treatment strategies but will not address the consequences of ceramide accumulation and SPL product deficiency.

Gene or enzyme replacement and gene editing are the only strategies with the potential to fully restore SPL activity in all patients with SPLIS. Developing an effective enzyme replacement therapy that can deliver SPL protein to the endoplasmic reticulum or provide a surrogate membrane system would be challenging. 
A

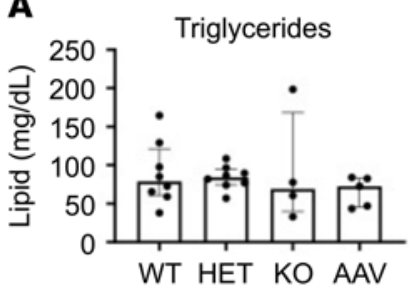

C

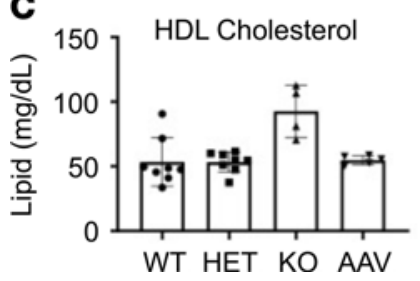

B

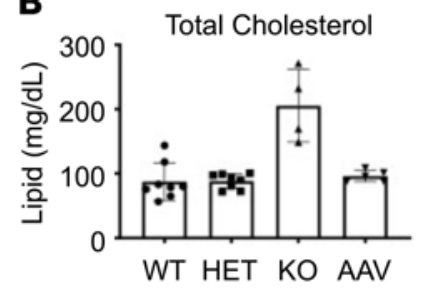

D

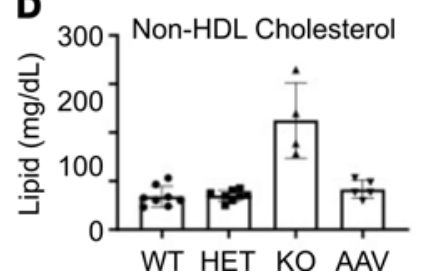

Figure 8. Plasma lipids of treated and untreated Sgpl1-KO mice. Plasma (A) triglycerides, (B) total cholesterol, (C) HDL-cholesterol, and (D) non-HDL-cholesterol (LDL, IDL, and VLDL) were measured in plasma of WT $(n=8)$, heterozygous (HET) $(n=8), \mathrm{KO}(n=4)$, and AAV-SPL-treated KO (AAV) mice, $(n=5)$. All mice were euthanized at $28 \mathrm{DOL}$. For triglycerides, 1-way ANOVA was applied, and there were NSDs between any of the groups. For all other lipids, 1-way ANOVA was used to compare WT, HET, and AAV-SPL, and unpaired $t$ test was used to compare KO and WT. Comparing KO vs. WT: (B, $P=0.0006) ;(\mathbf{C}, P=0.007) ;(\mathbf{D}, P=0.0002)$. There are NSDs between WT, HET, and AAV-SPL for any of the lipids.

While gene editing technology is developing rapidly, problems with off-target effects and efficient delivery in an intact organism remain to be solved. Based on these considerations and the lack of an available mouse model for testing gene editing approaches, we chose to investigate gene therapy as a promising near-term therapeutic strategy for SPLIS, a condition in which the risk/benefit ratio is likely to be acceptable.

We delivered human SGPL1 via AAV-mediated gene transfer to newborn Sgpl1-KO pups, using the strong, constitutive CMV promoter/enhancer to drive expression of the transgene. The dose was chosen based on the concentration of our viral preparation and the maximum tolerated volume of an i.v. bolus that newborn pups can safely tolerate (i.e., about $20 \mu \mathrm{L}$ ). We chose to investigate the impact of our treatment on survival as a first goal, with the expectation that a dose-response experiment might be necessary to optimize any discernable impact on survival. The effects we observed in the first 10 pups were so dramatic — with one-third of the cohort living 8 to 11 months - that we were compelled to undertake further analysis of the effects of our treatment on key disease features and lipids to uncover the reasons for this auspicious outcome.

After optimization of our injection protocol, most treated mice survived for months without developing nephrosis, as shown by normal urine ACR and plasma albumin levels and no evidence of glomerulosclerosis. This would suggest that $7 \times 10^{14} \mathrm{vg} / \mathrm{kg}$ represents a threshold dose needed to achieve efficacy in our model.

The STAT3 pathway was activated in the kidney parenchyma of Sgpl1-KO mice, as shown by Stat3 phosphorylation, upregulation of Stat3 target genes, and upregulation of Il-6. Importantly, this proinflammatory phenotype was prevented by treatment with AAV-SPL but not by a catalytically inactive control. STAT3 transcriptional activation has been implicated in the pathogenesis of diabetic kidney disease, Alport syndrome, lupus nephritis, nephrotoxic nephritis, and polycystic kidney disease (34). In addition, STAT3 activation has been shown to promote the progression of focal segmental glomerulosclerosis, which is manifest in patients with SPLIS (46-48). One of the many activators of the Stat3 pathway is S1PR-mediated signaling $(30,31,49)$. We found previously that gut-specific disruption of murine Sgpl1 results in sensitivity to colitis due to Stat3 activation (50). Thus, we suspected activation of Stat3 and resulting inflammation might underlie the nephrotoxic consequences of global Sgpl1 disruption. Our findings suggest that STAT3 represents a critical nexus between S1P signaling and kidney injury. While beyond the scope of this study, further investigation of the role of STAT3 in SPLIS and the intersection of sphingolipids, STAT3, and kidney disease is clearly warranted.

A battery of neurobehavioral tests designed to measure the normal acquisition of key developmental milestones of murine pups from birth to weaning was used to compare the developmental progress of untreated and treated Sgpl1-KO pups and WT littermate controls. Our results revealed the delayed achievement of 6 milestones in untreated KO pups, consistent with the neurological and behavioral defects observed 
Table 1. Liver and kidney function, glucose, and electrolytes

\begin{tabular}{|c|c|c|c|c|c|c|c|c|c|c|}
\hline $\begin{array}{l}\text { Genotype/ } \\
\text { Treatment }\end{array}$ & $\begin{array}{l}\text { ALT U/L } \\
(0-403)^{A}\end{array}$ & $\begin{array}{l}\text { AST U/L } \\
(0-552)^{A}\end{array}$ & $\begin{array}{l}\text { Total Bili mg/ } \\
\text { dL }(0.0-0.2)^{A}\end{array}$ & $\begin{array}{c}\text { Albumin g/ } \\
\text { dL }(2.9-4.0)^{\mathrm{A}}\end{array}$ & $\begin{array}{l}\text { BUN mg/dL } \\
(15.2-34.7)^{A}\end{array}$ & $\begin{array}{c}\text { Creat mg/dL } \\
(0.0-0.3)^{\mathrm{A}}\end{array}$ & $\begin{array}{l}\text { Glc mg/dL } \\
(130-254)^{A}\end{array}$ & $\begin{array}{c}\text { Na mmol/L } \\
(150-160)^{A}\end{array}$ & $\begin{array}{c}\text { Phos mg/dL } \\
(7.5-10.7)^{\mathrm{A}}\end{array}$ & $\begin{array}{l}\mathrm{Cl} \mathrm{mmol} / \mathrm{L} \\
(105-118)^{\mathrm{A}}\end{array}$ \\
\hline WT $(n=4)$ & $31.5 \pm 14.7$ & $69.3 \pm 22$ & $0.02 \pm 0.01$ & $3.5 \pm 0.4$ & $24.2 \pm 4.7$ & $0.1 \pm 0.02$ & $290 \pm 57$ & $151 \pm 13$ & $7.2 \pm 2.9$ & $111 \pm 10$ \\
\hline $\operatorname{HET}(n=4)$ & $112.6 \pm 58.7$ & $148 \pm 56$ & $0.06 \pm 0.07$ & $3.2 \pm 0.1$ & $22.3 \pm 0.3$ & $0.1 \pm 0.02$ & $208 \pm 36$ & $145 \pm 1$ & $10.6 \pm 1.6$ & $106 \pm 1$ \\
\hline $\begin{array}{l}\mathrm{WT}+\mathrm{HET} \\
(n=8)\end{array}$ & $71.6 \pm 58.8$ & $108.6 \pm 57.7$ & $0.04 \pm 0.05$ & $3.3 \pm 0.3$ & $23.2 \pm 3.2$ & $0.1 \pm 0.02$ & $249 \pm 62$ & $148 \pm 9$ & $8.9 \pm 2.8$ & $108 \pm 7$ \\
\hline $\mathrm{KO}(n=4)$ & $129.3 \pm 21.8$ & $387.1 \pm 136.7$ & $0.13 \pm 0.18$ & $1.5 \pm 0.7$ & $85 \pm 51.9$ & $0.18 \pm 0.08$ & $132 \pm 31$ & $148 \pm 8$ & $10.9 \pm 1.2$ & $116 \pm 2$ \\
\hline $\begin{array}{c}P \text { value } \\
\text { (WT+HET } \\
\text { vs. AAV) }\end{array}$ & NS & NS & NS & NS & 0.005 & 0.01 & NS & NS & NS & 0.05 \\
\hline $\begin{array}{c}P \text { value } \\
\text { (WT+HET }\end{array}$ & NS & 0.007 & NS & 0.005 & 0.003 & 0.02 & 0.04 & NS & NS & NS \\
\hline
\end{tabular}

ALaboratory reference ranges for adult BALB/c mice, not sex specific or age specific. ALT, alanine aminotransferase; AST, aspartate transaminase; Bili, bilirubin; BUN, blood urea nitrogen, Creat, creatinine, Glc, glucose; Phos, phosphorus.

in brain-specific Sgpl1-KO mice (26). In contrast, treated KO pups showed no evidence of developmental delay. A detailed study to monitor motor and behavioral function of treated Sgpl1-KO mice that reach adulthood will be required to establish the full impact of AAV-SPL on neuronal function. Optimization of brain targeting, such as by intrathecal or intracerebral delivery or using other capsid types that efficiently target neurons, may be required to maximize the impact of treatment on the CNS.

Our results show for the first time to our knowledge that Sgpl1-KO pups exhibit corticosterone deficiency. Consistent with a state of glucocorticoid insufficiency, plasma glucose levels of KO pups were at the low end of the normal range and significantly lower $(P=0.04)$ than those of WT pups. While plasma ACTH levels trended lower in KO compared to WT mice, these trends were not statistically significant. Further study will be required to fully characterize the adrenal cortical defect, the hypothalamic-pituitary-adrenal axis, and the function of other endocrine organs to establish the origin and extent of the endocrine defect. Treatment with AAV-SPL did not prevent corticosterone deficiency in our study. Interestingly, the relative hypoglycemia of the KO pups was corrected with AAV-SPL treatment. This finding suggests the possibility that glucocorticoid deficiency was in fact corrected by treatment but that we failed to detect this because of a sampling error or an insufficient number of subjects in our analysis.

Additional phenotypes reported in Sgpl1-KO pups, including anemia, high circulating cholesterol levels, and high Th-17 and other cytokines, were confirmed in our study. In addition, we observed elevated levels of the profibrogenic cytokine Tgf- $\beta$ in Sgpl1-KO mouse tissues. These phenotypes were reduced or completely prevented by AAV-SPL treatment. The complete responsiveness of hypercholesterolemia to AAV-SPL may be due to the high transduction efficiency of the liver with AAV9 combined with the liver representing the main site of cholesterol biosynthesis. On the other hand, lymphopenia persisted in treated $\mathrm{KO}$ mice, consistent with the known sensitivity of lymphocyte trafficking to modest increases in circulating S1P. While this is a limitation of our treatment, immunological rescue may not be necessary. Despite lymphopenia in patients with SPLIS, T cell function persists, and response to childhood vaccines is usually present $(3,10)$. AAV-SPL-treated $\mathrm{KO}$ mice mounted an immune response to both AAV and the transgene, indicating their immune system is functional despite their lymphopenia. Similarly, mice lacking SPL specifically in bone marrow are healthy, with normal life spans despite their lymphopenia (17). If necessary, systemic gene therapy could be combined with bone marrow transplantation, as has been reported for other genetic disease models (51).

The high tissue levels of S1P and upstream sphingolipid intermediates in the livers of untreated Sgpl1KO pups were substantially reduced in response to treatment, although the levels remained well above those of WT controls. Similarly, plasma S1P and other circulating sphingolipids were at least 50\% lower in treated versus untreated $\mathrm{KO}$ mice. These findings demonstrate that tissue SPL activity in response to AAVSPL treatment markedly attenuated the biochemical derangements characteristic of SPLIS. 
A

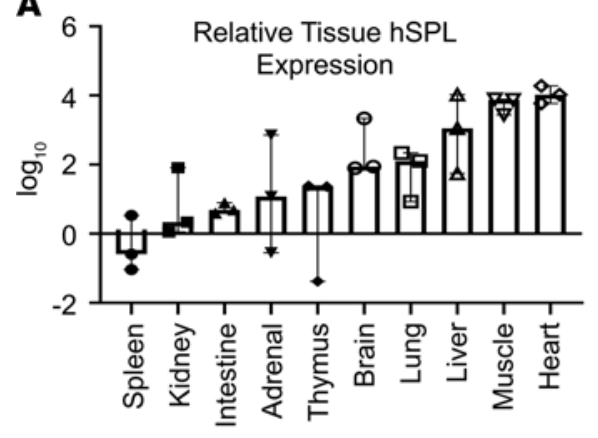

B

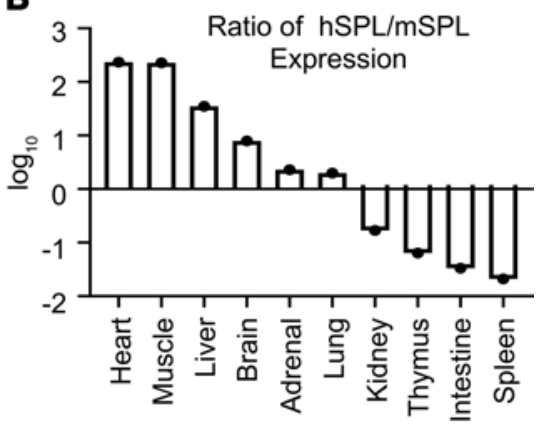

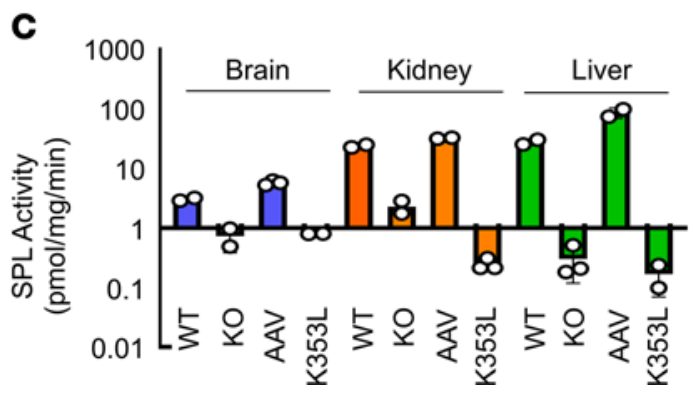

Figure 9. Bioavailability of AAV-SPL based on SPL gene expression and enzyme activity. (A) Relative hSPL (SCPL1) levels in different tissues of AAV-SPL-treated KO mice, shown as $\log _{10}$ values. (B) Ratio of hSPL (SCPL1) to mSPL (Sgpl1) in tissues of WT and AAV-SPL-treated KO mice, respectively. (C) SPL activity levels in brain (blue bars), kidney (red bars), and liver (green bars) of WT, KO, AAV-SPL-treated KO (AAV) and AAV-SPL ${ }^{\text {K353L }}$ treated KO (K353L) mice. Student's $t$ test: in all 3 tissues, for WT vs. KO, $P<0.03$; AAV vs. KO, $P<0.02$; KO vs. K353L, NSD. For A-C, $n=3$ per group.

Human SPL was widely expressed in treated $\mathrm{KO}$ mice, being detectable in both tubular and glomerular cells of the kidney, medullary cells of the adrenal gland, and Purkinje cells of the cerebellum. The transgene was expressed at $17 \%$ of endogenous levels in the kidney, but this may suffice, considering that podocytes, the key cells responsible for the blood filtration function of the kidney, make up only $3 \%$ of total kidney mass. The highly specific pattern of hSPL expression in cerebellar Purkinje cells mirrored that of endogenous mSPL, which is unexpected from a CMV promoter-driven transgene but can be explained by the coincidental Purkinje cell-specific cerebellar expression pattern of AAV9 after i.v. delivery to newborn mice (38). Importantly, expression in liver persisted for up to 11 months in long-lived mice, demonstrating the durability of the effect. The expression pattern was consistent with the effect on specific phenotypes, including protection against nephrosis, neurological defects, and inflammatory signaling in the liver. Use of genetically engineered capsids may further enhance site-specific and temporal transgene expression. For example, rationally designed artificial capsids such as Anc80 and PHP.eB may improve delivery to kidney and neuronal tissues, respectively $(39,52)$.

There are several limitations to our study. A single AAV-SPL dose was used in the entire experiment, so we cannot identify a minimal effective dose. We chose AAV9 to package the virus because of its broad tropism. Despite our promising bioavailability results, the potential for different capsids to achieve still higher expression remains. We chose the first days of life as a single treatment time point. It remains possible that administration of virus at a later age, but still before irreversible organ damage occurs, would increase transduction efficiency and efficacy.

Our analysis of neurodevelopment was hampered by the inability of the operator to maintain blindness to genotype because untreated Sgpl1-KO pups are runted, which becomes obvious after the first week of life. Reliance on highly objective milestones along with the presence of 2 observers at each testing period were employed to minimize observer bias.

Anti-AAV antibodies were detected in treated mice. Their presence could present challenges to optimization and translation to the clinic, considering that $30 \%$ of patients have anti-capsid 9 antibodies, causing them to be excluded from clinical trials with AAV9. Further, gene therapy could elicit an immune response to the transgene, especially in patients with SPLIS who produce no SPL protein. These challenges could be overcome by using endopeptidase to eliminate capsid-specific antibody, as recently described (53). 


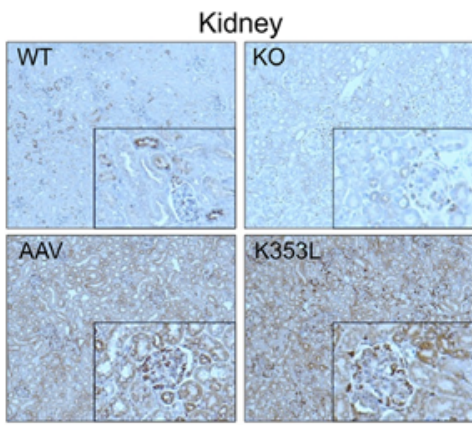

Adrenal
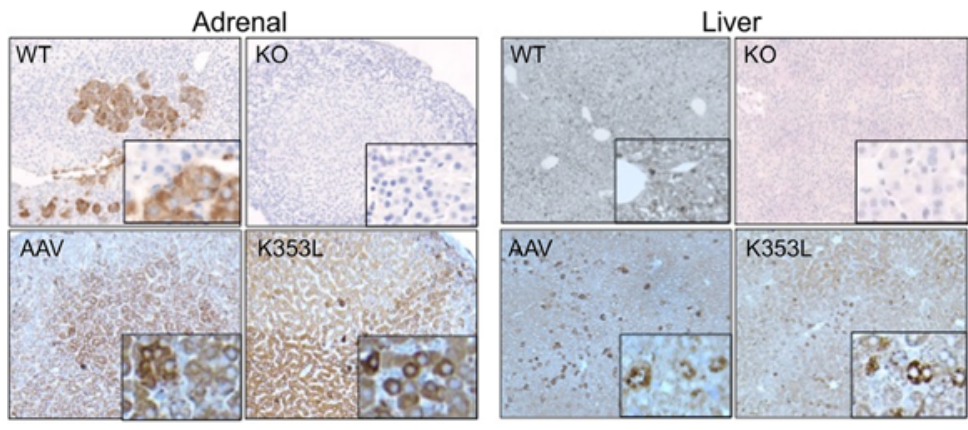

Figure 10. Expression pattern of $\mathrm{mSPL}$ and $\mathrm{hSPL}$ in murine tissues. IHC was performed on fixed tissue sections

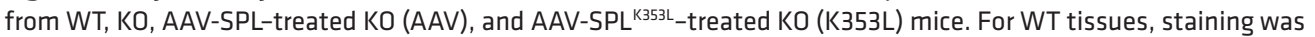
performed using anti-mSPL. For all other groups, staining was performed with anti-hSPL. KO tissues were also stained with anti-mSPL and were negative for signal (data not shown). Brain tissues from KO mice treated with PHP.eB-hSPL are shown in comparison with AAV9-hSPL. Insets show enlarged image detail for each quadrant to highlight cells with positive signal; original magnification, $\times 20$.

The high ectopic expression of SPL we achieved by expressing SGPL1 under the control of the CMV promoter could have undesirable effects, such as the susceptibility of the CMV regulatory sequence to silencing, potential integration effects including carcinogenesis, and toxicity of ectopic SPL expression. Substituting an alternate promoter could lead to improved efficacy, bioavailability, and safety compared with the CMV promoter.

We included both sexes in our study, and both male and female mice were among the longest lived animals. However, a larger study will be required to discern any potential sex-specific differences in efficacy and effects on male and female reproduction.

Last, the Sgpl1-KO mouse - while a robust model of severe SPLIS - may not faithfully emulate the pathologies found in SPLIS forms caused by mutations that do not eliminate SPL entirely. In the future, the development of murine models harboring missense mutations created through gene editing technologies would afford additional systems for testing therapeutic strategies employing gene replacement and repair.

In aggregate, our findings demonstrate that AAV-SPL treatment delivered early in life can avert the development of nephrosis, neurobehavioral deficits, lipidosis, and aberrant inflammatory signaling, enabling long-term survival in a mouse model of SPLIS. The efficacy of AAV-SPL is consistent with the broad expression pattern and enzyme activity observed in mice treated with AAV-SPL. Our preclinical results suggest that SGPL1 gene replacement holds promise as a universal targeted treatment for SPLIS. Safety studies and human clinical trials will ultimately reveal the translational relevance of our auspicious initial results.

\section{Methods}

Reagents. We used S1P, hexadecenal(d5), and Ceramide/Sphingoid Internal Standard Mixture I (Avanti Polar Lipids); 2-hydrazinoquinoline (MilliporeSigma); and HPLC vials (Filtrous).

Vector production and packaging. Human WT SGPL1 cDNA, hSPL ${ }^{\mathrm{K} 353 \mathrm{~L}} \mathrm{cDNA}$, and hSPL-tRFP were separately subcloned into EcoRI/XhoI sites in pAAV-MCS (Agilent Technologies). Inserts were confirmed by DNA sequencing. AAV was packaged with different capsids to generate AAV8-, AAV9-, and AAV-PHP. eB-SPL virus. AAV was packaged using an adenovirus-free system in which AAV-SPL, pHelper, and AAV-RC plasmid pUCmini-iCAP-PHP.eB (Addgene plasmid 103005) were cotransfected into HEK293 cells (ATCC). Virus was harvested and purified via iodixanol gradient ultracentrifugation (54). After validating activity, 
Table 2. S1P levels in Sgpl1 WT, HET, and KO organs (pmol/mg)

\begin{tabular}{ccccc}
\hline Organ & WT $(\boldsymbol{n}=\mathbf{3})$ & HET $(\boldsymbol{n}=\mathbf{3})$ & KO $(\boldsymbol{n}=\mathbf{3})$ & $\begin{array}{c}\text { KO/WT } \\
\text { (fold change) }\end{array}$ \\
\hline Intestine & 4.93 & 3.23 & 1338.01 & 271 \\
\hline Stomach & 85.3 & 55.36 & 545.16 & 6 \\
\hline Heart & 4.82 & 14.11 & 2088.87 & 433 \\
\hline Lung & 33.29 & 35.47 & 2889.36 & 87 \\
\hline Spleen & 19.43 & 27.3 & 2267.11 & 117 \\
\hline Muscle & 4.11 & 5.02 & 320.82 & 78 \\
\hline Brain & 43.55 & 26.21 & 74.25 & 1.7 \\
\hline Liver & 3.73 & 2.93 & 1137.7 & 305 \\
\hline Thymus & 13.01 & 4.26 & 1172.5 & 90 \\
\hline Kidney & 25.91 & 8.33 & 2416.47 & 93 \\
Colon & 10.41 & 15.23 & 586.43 & 56 \\
\hline
\end{tabular}

AAV9-hSPL, PHP.eB-hSPL, and AAV9-hSPL ${ }^{\mathrm{K} 353 \mathrm{~L}}$ virions were prepared at large scale for in vivo use. Virus titers were quantified by Taqman quantitative PCR, and probes were determined to be $\sim 3.5 \times 10^{10} \mathrm{vg} / \mu \mathrm{L}$.

Functional testing. Virus particles expressing WT hSPL, hSPL, and RFP, or catalytically inactive mutant hSPL ${ }^{\text {K333L }}$, were used to infect immortalized fibroblasts derived from a patient with SPLIS (10). Fibroblasts were generated and cultured in DMEM with $10 \%$ FBS as described previously (55). AAV9-hSPL and AAV9hSPL-tRFP viruses were used to transduce fibroblasts. After 48 hours, cells were harvested for analysis.

Immunoblotting. Proteins were extracted as described (10). Antibodies used were goat anti-human SGPL1 (AF5535, R\&D Systems, Bio-Techne), anti-mouse Sgpl1 (56), rabbit anti-GAPDH (sc-25778, Santa Cruz Biotechnology), anti-phosphorylated Stat3 (9145, Cell Signaling Technology), and anti-total Stat3 (9131, Cell Signaling Technology). HRP-conjugated secondary antibodies [115-035-003, Goat Anti-Mouse IgG (H+L); 111-035-144, Goat Anti-Rabbit IgG (H+L), Jackson ImmunoResearch; sc-2020, donkey antigoat IgG-HRP, Santa Cruz Biotechnology] were used to detect signal using SuperSignal West Pico kit (Thermo Fisher Scientific). Radiographic bands were quantified using NIH ImageJ.

SPL assays. SPL activity was quantified by measuring formation of (2E)-hexadecenal(d5) by quantification of its hydrazine derivative as described (57).

Animals. SPL-KO mice, bred for over a decade in the UCSF animal facility, exhibit no SPL expression or activity and accumulate sphingolipids (15). The KO allele was generated using the ROSAFARY gene trap vector $(24,58)$. Heterozygous mice are viable and fertile and are crossed to obtain homozygous progeny. The $\mathrm{KO}$ is born at $25 \%$ frequency. The line was backcrossed to C57BL/ 6 background for 10 generations. Sgpl1 heterozygous $\mathrm{KO}$ mice were maintained in an Association for Assessment and Accreditation of Laboratory Animal Care International-accredited animal facility with an automated light/dark cycle of $7 \mathrm{am} / 7 \mathrm{pm}$. Pups were genotyped by toe biopsy using primers: SPL-F: CGCTCAGAAGGCTCTGAGTCATGG, SPL wt-R: CCAAGTGTACCTGCTAAGTTCCAG, and SPL ko-R: CATCAAGGAAACCCTGGACTACTG.

$A A V$ treatments. Homozygous $\mathrm{KO}$ pups were anesthetized with isoflurane and injected with virus (3.5$7.0 \times 10^{11} \mathrm{vg}$ ) in $10-20 \mu \mathrm{L}$ sterile saline into the superficial temporal vein using a 33-gauge needle and Yale model YA-12 syringe pump. To aid temporal vein injection, the vector solution contained $1 \%$ food coloring. In some cases, virus solution was instead injected into the liver parenchyma as described (59). Injected mice were monitored and weighed every 3 days and subjected to 24-hour urine collections in a metabolic chamber, serial neurobehavioral examinations, and/or phlebotomy at various time points. Survival study mice were euthanized when they reached the humane endpoint. Other mice were euthanized at 21-28 DOL.

Laboratory analyses. Serum and urine albumin and creatinine were measured using the COBAS INTEGRA 400 plus instrument (Roche Diagnostics) by the University of California Davis Comparative Pathology Laboratory (Davis, California, USA).

Kidney pathology. Periodic acid-Schiff staining of $3 \mu \mathrm{M}$ sections of FFPE mouse kidneys was performed. Glomerular tuft area (which includes capillaries, mesangium, and podocytes) was measured across 30 glomeruli from each mouse kidney, sampling the full depth of the cortex, using QuPath quantitative pathology and bioimage analysis software after scanning sections stained with Periodic acid-Schiff to whole-slide images with a Leica AT2 scanner (Aperio). 

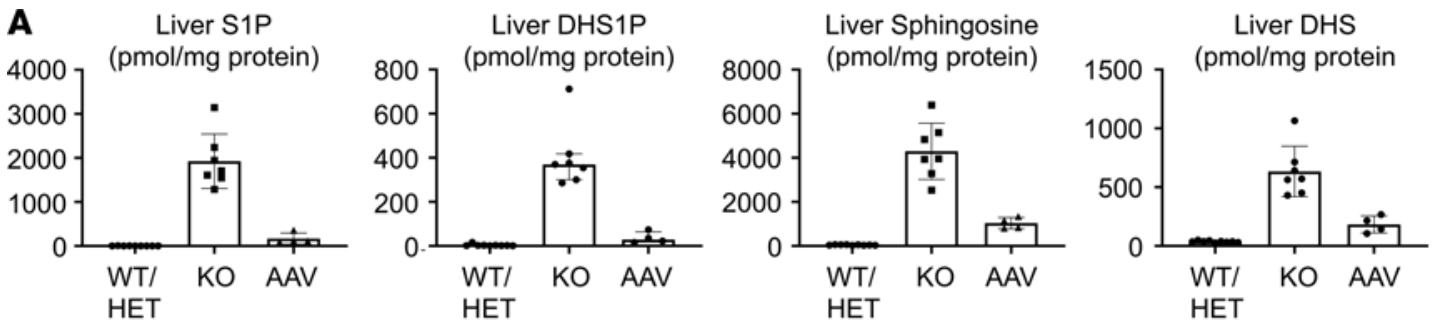

B

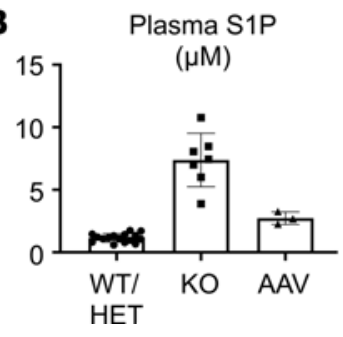

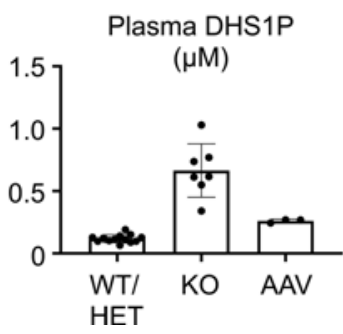

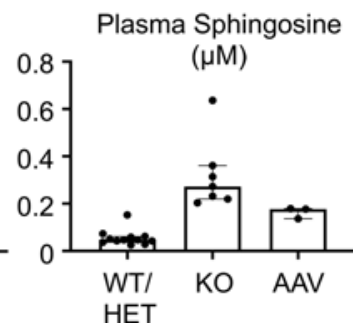

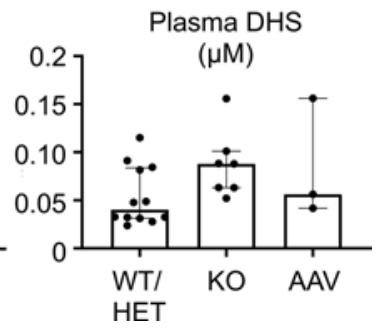

Figure 11. Attenuation of sphingolipid accumulation in AAV-SPL-treated Sgpl1-KO mice. S1P, dihydrosphingosine-1-phosphate (DHS1P), sphingosine, and dihydrosphingosine (DHS) in the (A) liver and (B) plasma of WT $(n=9), \mathrm{KO}(n=7)$, and AAV-SPL-treated KO (AAV, $n=4)$ mice. For liver S1P, liver DHS1P, and plasma sphingosine, Mann-Whitney $U$ with Bonferroni's correction was applied. For liver sphingosine, liver DHS, plasma S1P, and plasma DHS1P, unpaired $t$ test with Bonferroni's correction was applied, and for liver DHS and plasma DHS1P, Welch's correction was additionally applied. For plasma DHS, Kruskal-Wallis was applied. For liver S1P: KO vs. WT/HET, $P<0.004$; AAV vs. WT/HET, $P=0.0058$; AAV vs. KO, $P=0.012$. For liver sphingosine: KO vs. WT/HET, $P<0.0002$; AAV vs. WT/HET, $P<0.0002$; AAV vs. KO, $P=0.0016$. For liver DHS1P: KO vs. WT/HET, $P=0.0004 ; \mathrm{AAV}$ vs. WT/HET, $P=0.0112 ; \mathrm{AAV}$ vs. KO, $P=$ 0.0122 . For liver DHS: KO vs. WT/HET, $P=0.0006 ; \mathrm{AAV}$ vs. WT/HET, $P=0.0062 ; \mathrm{AAV}$ vs. KO, $P=0.049$. For plasma S1P: KO vs. WT/HET, $P=0.0002 ; \mathrm{AAV}$ vs WT/HET, $P=0.0002$; AAV vs. KO, $P=0.014$. For plasma sphingosine: KO vs. WT/HET, $P<0.0002$; AAV vs. WT/HET, $P<0.0018 ; A A V$ vs. KO, $P=0.033$. For plasma DHS1P: KO vs. WT/HET, $P=0.001$; AAV vs. WT/HET, $P<0.0002$; AAV vs. KO, $P=0.0048$. For plasma DHS: there were NSDs in any of the groups.

RNA isolation and $m R N A$ analysis. Total RNA was extracted by TRIzol reagent (Thermo Fisher Scientific). DNase was used to remove genomic DNA contamination as described (60). A total of $4 \mu \mathrm{g}$ of DNase Itreated RNA was used for the first-strand cDNA synthesis by using the SuperScript III reverse transcriptase to synthesize reverse transcription products (Life Technologies, Thermo Fisher Scientific). Quantitative realtime PCR was performed by using PowerUp SYBR Green Master Mix in QuantStudio 12K Flex Real-Time PCR System (Life Technologies, Thermo Fisher Scientific). Primers used are listed in Supplemental Table 1.

Neurological examination of preweaned mice. A battery of neurodevelopmental tests for preweaned mice was developed in accordance with existing protocols (61-64). Mice were assessed for achievement of bilateral eye opening starting on DOL 11, hearing onset starting on DOL 11, adult walking pattern starting on DOL 3 grip strength starting on DOL 21, and cliff aversion reflex starting on DOL 3. The walking pattern was also evaluated on DOL 9, and pups were given a score of 0 for nonlinear pivoting, 1 for mixed ambulation, 2 for fully linear movement. Each mouse underwent 3 sets of grip strength tests using a Chatillon Ametek digital force gauge model DFIS-10. Cliff aversion reflex was marked as present or absent daily across 8 days of testing. A score was assigned to each pup by summing the number of days that the reflex was present, resulting in a score from 0 to 8 . Results were aggregated across the 4 groups. Two operators were present at each testing session.

Corticosterone and ACTH measurements. Corticosterone was measured by radioimmune assay (RIA), using a commercial kit (MP Biomedicals) at the University of Virginia Ligand Assay \& Analysis Core (Charlottesville, Virgina, USA). The method was validated for mouse serum using a protocol based on recommendations of the Endocrine Society's Sex Steroid Assays Reporting Task Force (65). The RIA evaluation included the following indices: accuracy, linearity, functional sensitivity, precision, and correlation to a previous or established method. Assay characteristics were as follows: sensitivity $=15 \mathrm{ng} / \mathrm{mL}$; intra-assay coefficient of variation $(\mathrm{CV})=6.3 \%$; inter-assay $\mathrm{CV}=7.1 \%$. ACTH was measured using the Abcam Mouse/Rat ACTH ELISA Kit, according to the manufacturer's instructions.

Cholesterol measurements. Plasma triglycerides, total cholesterol, and HDL-cholesterol were measured by enzymatic endpoint analysis using enzyme reagent kits (AMS Alliance) on a clinical chemistry analyzer (Liasys 330, AMS Alliance).

IHC. IHC was performed essentially as described (37). FFPE tissues were deparaffinized and incubated for 30 minutes in 3\% hydrogen peroxide/methanol to quench endogenous peroxidases. Sections were rinsed 
A

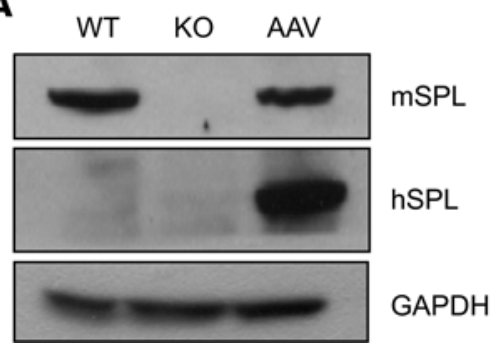

B

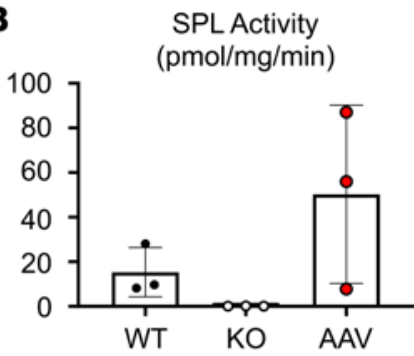

Figure 12. Durability of AAV-SPL. (A) Abundance of liver mSPL and hSPL are shown by immunoblot of livers from WT, KO, and long-lived AAV-SPL-treated KO mice (AAV). Blots were probed using anti-mSPL, which detects mSPL and crossreacts with hSPL, and using anti-hSPL, which is specific for hSPL. WT liver expresses mSPL but not hSPL. Untreated $\mathrm{KO}$ does not express either protein. Liver of the AAV-SPL-treated KO mouse euthanized at 11.5 months of age shows abundant hSPL, detected by both antibodies. GAPDH is a loading control. (B) SPL activity measured in livers of WT, KO, and long-lived AAV-SPL-treated KO (AAV) mice ( $n=3 /$ group) euthanized at 11 months of age. Using unpaired $t$ test with Bonferroni's correction, there were NSDs between the groups.

in PBS and immunostained with anti-mSPL antisera at 1:200 dilution in $0.5 \% \mathrm{PBS} / \mathrm{OVA}$ at $37^{\circ} \mathrm{C}$ for 1 hour after antigen retrieval with citrate buffer $\left(\mathrm{pH}\right.$ 6.0) in an autoclave set for $125^{\circ} \mathrm{C}$ for 2 minutes; slides were cooled for 1 hour before adding secondary antibody. Secondary antibody was biotinylated anti-rabbit (PV6121, Leica Biosystems) diluted 1:1000 in 0.5\% PBS/OVA and incubated for 30 minutes at room temperature. Sections were incubated with VECTASTAIN Elite ABC kit (Vector Laboratories, Maravai LifeSciences) for $30 \mathrm{~min}$ utes and rinsed in PBS. Detection was performed with DAB (Vector Laboratories, Maravai LifeSciences) for 2 minutes and counterstained in hematoxylin.

Sphingolipid profiling. Plasma was obtained from anticoagulated whole blood by subjecting it to centrifugation at about $250 \mathrm{~g}$ for 10 minutes at $4^{\circ} \mathrm{C}$, followed by collection of plasma, which was stored at $-80^{\circ} \mathrm{C}$ until analysis. To extract lipids, frozen tissues were bead homogenized in Tris buffer using a FastPrep FP120 cell disrupter (Thermo Fisher Scientific). Plasma and tissue homogenates were spiked with internal standard and extracted as described (66). Internal standard mixture contained sphingosine, C17:0 ceramide, D-erythro-sphingosine-d7-1-P, C12:0 ceramide-1-P, sphingomyelin (d18:1/C12:0), and lyso-sphingomyelin (d17:1) (Avanti Polar Lipids). Targeted metabolomics was performed using single reaction monitoring with an Agilent Technologies 6490 triple-quadrupole LC-MS/MS instrument coupled with a 1290 Infinity UPLC system as described (66).

Detection of antibodies by ELISA. For detection of anti-AAV9, $17 \mu \mathrm{L}$ of AAV at $1.17 \times 10^{13} \mathrm{vg} / \mathrm{mL}$ was added to $10 \mathrm{~mL}$ of coating buffer (100 $\mathrm{mM}$ carbonate/bicarbonate buffer containing $3.03 \mathrm{~g} \mathrm{Na}_{2} \mathrm{CO}_{3}, 6.0 \mathrm{~g}$ $\mathrm{NaHCO}_{3}$ per liter, $\mathrm{pH}$ 9.6). A total of $100 \mu \mathrm{L}$ of this solution was used to coat each well of a 96-well plate (high-binding polystyrene Immulon $2 \mathrm{HB}$ plate). The plates were left at $4^{\circ} \mathrm{C}$ overnight and the next day were washed 4 times with $200 \mu \mathrm{L}$ TBS-Tween 20 (TBST) per well. Next, $200 \mu \mathrm{L}$ of $\%$ BSA in TBST was used to block the wells overnight at $4^{\circ} \mathrm{C}$, followed by washing. Anti-AAV9 mouse monoclonal antibody (MilliporeSigma clone HL2372, catalog MABF2309) was used to establish a standard curve. Plasma from 3 untreated and 3 AAV-SPL-treated mice was diluted 1:100 and 1:500, and $100 \mu \mathrm{L}$ of each dilution was added to each well in triplicate. All dilutions were done in $2 \%$ BSA in TBST. Incubation was at $4{ }^{\circ} \mathrm{C}$ overnight followed by washing. The following day, $100 \mu \mathrm{L}$ of 1:4000 dilution of an alkaline phosphatase-conjugated goat anti-mouse antibody (115-055-003, Jackson ImmunoResearch Laboratories) diluted in 2\% BSA in TBST was added to each well. The plate was incubated for 1 hour at room temperature, then washed 4 times with TBST. A solution of $1 \mathrm{mg} / \mathrm{mL}$ of pNPP-disodium hexahydrate (bioWORLD 21530110-4) made in a buffer containing $100 \mathrm{mM}$ Tris at $\mathrm{pH} 9.5,100 \mathrm{mM} \mathrm{NaCl}$, and $5 \mathrm{mM} \mathrm{MgCl}_{2}$ was added at 100 $\mu \mathrm{L}$ per well to develop the ELISA at $37^{\circ} \mathrm{C}$ for $20-40$ minutes. A plate reader was used to measure the absorbance at $405 \mathrm{nM}$, and results are reported as $\mathrm{ng} / \mathrm{mL}$. For detection of anti-hSPL, $200 \mathrm{ng}$ of human SGPL1 recombinant protein (Abnova catalog H00008879-P01) was used to coat each well. The following day, $280 \mu \mathrm{L} /$ well of TBST containing $5 \%$ fat-free milk was used to block the plate overnight. Twenty hours later, plasma samples were diluted 1:100 in TBST containing $2 \%$ BSA, and $100 \mu \mathrm{L}$ was added to each of triplicate wells. Serial dilutions of polyclonal mouse anti-human SGPL1 antibody (Abnova catalog H00008879-A01) were added to separate wells as a positive control. The development of the ELISA plate was as for anti-AAV9 detection. Anti-hSPL is reported in arbitrary units. 
Statistics. For comparison of 2 groups with normally distributed data, an unpaired $t$ test was performed. For variances with $P<0.05$, Welch's correction was applied. For comparison of more than 2 groups with normally distributed data, 1-way ANOVA was performed. For skewed data, the Mann-Whitney $U$ test was applied when 2 groups were compared, and Kruskal-Wallis was applied when more than 2 groups were compared. When multiple comparisons were made, Bonferroni's correction was performed. Two-tailed $t$ tests were performed in all cases. For all analyses, $P<0.05$ was considered significant. Normally distributed data are presented as mean \pm standard deviation. Skewed data are represented as median \pm the interquartile range.

Study approval. Human skin fibroblasts were generated from skin biopsy samples obtained with informed consent in accordance with a Benioff Children's Hospital Oakland-approved IRB protocol. Written informed consent was received from participants prior to inclusion in the study. All mouse studies were conducted in accordance with an approved University of California San Francisco IACUC protocol.

\section{Author contributions}

JDS directed the study, conducted data analysis, and wrote the manuscript. PZ contributed to study design and performed most experiments and data analysis. GBT delivered AAV vectors to mice and performed phlebotomy and tissue harvest. GLW contributed to interpretation of results and provided critical insights. DPM and FT performed and analyzed quantitative RT-PCR studies. JYL performed SPL assays and sphingolipid measurements. JBH, JL, and YY performed and analyzed kidney pathology. BO performed immunoblotting and ELISA. JYW performed neurodevelopmental milestone assays. SK and RMK conducted cholesterol and triglyceride measurements. NK performed statistical analyses and data graphing.

\section{Acknowledgments}

This study was performed with support from NIH S10OD0018070, the Swim Across America Foundation, a UCSF Catalyst Award, a gift from James Shayman, a gift from KMD Foundation, and a gift from Ultragenyx Pharmaceutical Inc. (to JDS). University of Virginia Ligand Assay \& Analysis Core is supported by the Eunice Kennedy Shriver National Institute of Child Health and Human Development/NIH Grant R24HD102061. We would like to thank Jody L. Smith for providing pAAV-MCS, AAV2/8, AAV2/9, and pHelper plasmids.

Address correspondence to: Julie D. Saba, Department of Pediatrics, UCSF, 550 16th Street, Box 0110, San Francisco, California 94143, USA. Email: Julie.Saba@ucsf.edu.

1. Choi YJ, Saba JD. Sphingosine phosphate lyase insufficiency syndrome (SPLIS): a novel inborn error of sphingolipid metabolism. Adv Biol Regul. 2019;71:128-140.

2. Dunn TM, et al. A perilous path: the inborn errors of sphingolipid metabolism. J Lipid Res. 2019;60(3):475-483

3. Lovric S, et al. Mutations in sphingosine-1-phosphate lyase cause nephrosis with ichthyosis and adrenal insufficiency. $J$ Clin Invest. 2017;127(3):912-928.

4. Prasad R, et al. Sphingosine-1-phosphate lyase mutations cause primary adrenal insufficiency and steroid-resistant nephrotic syndrome. J Clin Invest. 2017;127(3):942-953.

5. Janecke AR, et al. Deficiency of the sphingosine-1-phosphate lyase SGPL1 is associated with congenital nephrotic syndrome and congenital adrenal calcifications. Hum Mutat. 2017;38(4):365-372.

6. Atkinson D, et al. Sphingosine 1-phosphate lyase deficiency causes Charcot-Marie-Tooth neuropathy. Neurology. 2017;88(6):533-542

7. Bamborschke D, et al. A novel mutation in sphingosine-1-phosphate lyase causing congenital brain malformation. Brain Dev. 2018;40(6):480-483

8. Settas N, et al. SGPL1 deficiency: a rare cause of primary adrenal insufficiency. J Clin Endocrinol Metab. 2019;104(5):1484-1490.

9. Linhares N, et al. Nephrotic syndrome and adrenal insufficiency caused by a variant in SGPL1. Clin Kidney J. 2018;11(4):462-467.

10. Zhao P, et al. Responsiveness of sphingosine phosphate lyase insufficiency syndrome to vitamin B6 cofactor supplementation. J Inherit Metab Dis. 2020;43(5):1131-1142.

11. Martin KW, et al. MRI spectrum of brain involvement in sphingosine-1-phosphate lyase insufficiency syndrome. $A J N R A m J$ Neuroradiol. 2020;41(10):1943-1948.

12. Kumar A, Saba JD. Lyase to live by: sphingosine phosphate lyase as a therapeutic target. Expert Opin Ther Targets. 2009;13(8):1013-1025

13. Cartier A, Hla T. Sphingosine 1-phosphate: lipid signaling in pathology and therapy. Science. 2019;366(6463):eaar5551.

14. Garris CS, et al. Sphingosine-1-phosphate receptor 1 signalling in T cells: trafficking and beyond. Immunology. 2014;142(3):347-353.

15. Bektas M, et al. Sphingosine 1-phosphate lyase deficiency disrupts lipid homeostasis in liver. J Biol Chem. 2010;285(14):10880-10889.

16. Schwab S, et al. Lymphocyte sequestration through S1P lyase inhibition and disruption of S1P gradients. Science. 2005;309(5741):1735-1739.

17. Zamora-Pineda J, et al. Dendritic cell sphingosine-1-phosphate lyase regulates thymic egress. J Exp Med. 2016;213(12):2773-2791.

18. Ikeda M, et al. Sphingosine-1-phosphate lyase SPL is an endoplasmic reticulum-resident, integral membrane protein with the pyridoxal 5'-phosphate binding domain exposed to the cytosol. Biochem Biophys Res Commun. 2004;325(1):338-343.

19. Weaver KN, et al. Sphingosine phosphate lyase insufficiency syndrome. In: Adam MP, et al., eds. GeneReviews. University of 
Washington; 2020.

20. Wang D, et al. Adeno-associated virus vector as a platform for gene therapy delivery. Nat Rev Drug Discov. 2019;18(5):358-378

21. Rabinowitz J, et al. Adeno-associated virus (AAV) versus immune response. Viruses. 2019;11(2):E102.

22. Srivastava A. In vivo tissue-tropism of adeno-associated viral vectors. Curr Opin Virol. 2016;21:75-80.

23. Keeler AM, Flotte TR. Recombinant adeno-associated virus gene therapy in light of luxturna (and Zolgensma and Glybera): where are we, and how did we get here? Annu Rev Virol. 2019;6(1):601-621.

24. Schmahl J, et al. PDGF signaling specificity is mediated through multiple immediate early genes. Nat Genet. 2007;39(1):52-60

25. Schumann J, et al. Reduced activity of sphingosine-1-phosphate lyase induces podocyte-related glomerular proteinuria, skin irritation, and platelet activation. Toxicol Pathol. 2015;43(5):694-703.

26. Mitroi DN, et al. Sphingosine 1-phosphate lyase ablation disrupts presynaptic architecture and function via an ubiquitin- proteasome mediated mechanism. Sci Rep. 2016;6:37064.

27. Allende M, et al. Sphingosine-1-phosphate lyase deficiency produces a pro-inflammatory response while impairing neutrophil trafficking. J Biol Chem. 2011;286(9):7348-7358.

28. Vogel P, et al. Incomplete inhibition of sphingosine 1-phosphate lyase modulates immune system function yet prevents early lethality and non-lymphoid lesions. PLoS One. 2009;4(1):e4112.

29. Yu H, et al. Revisiting STAT3 signalling in cancer: new and unexpected biological functions. Nat Rev Cancer. 2014;14(11):736-746.

30. Lee H, et al. STAT3-induced S1PR1 expression is crucial for persistent STAT3 activation in tumors. Nat Med. 2010;16(12):1421-1428.

31. Liu Y, et al. S1PR1 is an effective target to block STAT3 signaling in activated B cell-like diffuse large B-cell lymphoma. Blood. 2012;120(7):1458-1465.

32. Zhang H, et al. Podocyte-specific JAK2 overexpression worsens diabetic kidney disease in mice. Kidney Int. 2017;92(4):909-921.

33. Tao J, et al. JAK-STAT activity in peripheral blood cells and kidney tissue in IgA nephropathy. Clin J Am Soc Nephrol. 2020;15(7):973-982

34. Pace J, et al. Targeting STAT3 signaling in kidney disease. Am J Physiol Renal Physiol. 2019;316(6):F1151-F1161.

35. MacKenzie SM, et al. Analysis of the aldosterone synthase (CYP11B2) and 11ß-hydroxylase (CYP11B1) genes. Methods Mol Biol. 2017;1527:139-150.

36. Ge X, et al. Aldo-keto reductase 1B7 is a target gene of FXR and regulates lipid and glucose homeostasis. J Lipid Res. 2011;52(8):1561-1568.

37. Borowsky AD, et al. Sphingosine-1-phosphate lyase expression in embryonic and adult murine tissues. J Lipid Res. 2012;53(9):1920-1931.

38. Huda F, et al. Distinct transduction profiles in the CNS via three injection routes of AAV9 and the application to generation of a neurodegenerative mouse model. Mol Ther Methods Clin Dev. 2014;1:14032.

39. Dayton RD, et al. More expansive gene transfer to the rat CNS: AAV PHP.EB vector dose-response and comparison to AAV PHP.B. Gene Ther. 2018;25(5):392-400.

40. Matloubian M, et al. Lymphocyte egress from thymus and peripheral lymphoid organs is dependent on S1P receptor 1. Nature. 2004;427(6972):355-360.

41. Cellini B, et al. The chaperone role of the pyridoxal 5'-phosphate and its implications for rare diseases involving B6-dependent enzymes. Clin Biochem. 2014;47(3):158-165.

42. Hou ZS, et al. Pharmacoperone drugs: targeting misfolded proteins causing lysosomal storage-, ion channels-, and G protein-coupled receptors-associated conformational disorders. Expert Rev Clin Pharmacol. 2018;11(6):611-624.

43. Shayman JA. Targeting glucosylceramide synthesis in the treatment of rare and common renal disease. Semin Nephrol. 2018;38(2):183-192.

44. Biswas S, et al. Substrate reduction intervention by L-cycloserine in twitcher mice (globoid cell leukodystrophy) on a B6;CAST/ Ei background. Neurosci Lett. 2003;347(1):33-36.

45. Herr DR, et al. Sply regulation of sphingolipid signaling molecules is essential for Drosophila development. Development. 2003;130(11):2443-2453.

46. Liang Y, et al. Expression of JAKs/STATs pathway molecules in rat model of rapid focal segmental glomerulosclerosis. Pediatr Nephrol. 2009;24(9):1661-1671.

47. Sharma M, et al. Janus kinase 2/signal transducer and activator of transcription 3 inhibitors attenuate the effect of cardiotrophin-like cytokine factor 1 and human focal segmental glomerulosclerosis serum on glomerular filtration barrier. Transl Res. 2015;166(4):384-398.

48. Tao J, et al. JAK-STAT signaling is activated in the kidney and peripheral blood cells of patients with focal segmental glomerulosclerosis. Kidney Int. 2018;94(4):795-808.

49. Loh KC, et al. Sphingosine-1-phosphate enhances satellite cell activation in dystrophic muscles through a S1PR2/STAT3 signaling pathway. PLoS One. 2012;7(5):e37218.

50. Degagné E, et al. Sphingosine-1-phosphate lyase downregulation promotes colon carcinogenesis through STAT3-activated microRNAs. J Clin Invest. 2014;124(12):5368-5384.

51. Heldermon CD, et al. Therapeutic efficacy of bone marrow transplant, intracranial AAV-mediated gene therapy, or both in the mouse model of MPS IIIB. Mol Ther. 2010;18(5):873-880.

52. Ikeda Y, et al. Efficient gene transfer to kidney mesenchymal cells using a synthetic adeno-associated viral vector. $J$ Am Soc Nephrol. 2018;29(9):2287-2297.

53. Leborgne C, et al. IgG-cleaving endopeptidase enables in vivo gene therapy in the presence of anti-AAV neutralizing antibodies. Nat Med. 2020;26(7):1096-1101.

54. Zolotukhin S, et al. Recombinant adeno-associated virus purification using novel methods improves infectious titer and yield. Gene Ther. 1999;6(6):973-985.

55. Vangipuram M, et al. Skin punch biopsy explant culture for derivation of primary human fibroblasts. J Vis Exp. 2013 (77):e3779.

56. Reiss U, et al. Sphingosine-phosphate lyase enhances stress-induced ceramide generation and apoptosis. J Biol Chem. 2004;279(2):1281-1290.

57. Suh JH, et al. A facile stable-isotope dilution method for determination of sphingosine phosphate lyase activity. Chem Phys Lipids. 2016;194:101-109. 
58. Schmahl J, et al. The PDGF signaling pathway controls multiple steroid-producing lineages. Genes Dev. 2008;22(23):3255-3267.

59. Chandler RJ, Venditti CP. Adenovirus-mediated gene delivery rescues a neonatal lethal murine model of mut $(0)$ methylmalonic acidemia. Hum Gene Ther. 2008;19(1):53-60.

60. Zhao P, et al. Anoctamin 6 regulates C2C12 myoblast proliferation. PLoS One. 2014;9(3):e92749.

61. Feather-Schussler DN, Ferguson TS. A battery of motor tests in a neonatal mouse model of cerebral palsy. $J$ Vis Exp. 2016;117(117):53569

62. Brown RE, et al. Developing standardized behavioral tests for knockout and mutant mice. ILAR J. 2000;41(3):163-174.

63. Roubertoux PL, et al. Measuring preweaning sensorial and motor development in the mouse. Curr Protoc Mouse Biol. 2018;8(1):54-78.

64. Nguyen AT, et al. Neurodevelopmental reflex testing in neonatal rat pups. J Vis Exp. 2017;122(122):55261.

65. Wierman ME, et al. Editorial: the new instructions to authors for the reporting of steroid hormone measurements. J Clin Endocrinol Metab. 2014;99(12):4375.

66. Suh JH, et al. Sphingosine-1-phosphate signaling and metabolism gene signature in pediatric inflammatory bowel disease: a matched-case control pilot study. Inflamm Bowel Dis. 2018;24(6):1321-1334. 